\title{
CD98 expression modulates intestinal homeostasis, inflammation, and colitis-associated cancer in mice
}

\author{
Hang Thi Thu Nguyen, ${ }^{1}$ Guillaume Dalmasso, ${ }^{1}$ Leif Torkvist, ${ }^{2}$ Jonas Halfvarson, ${ }^{3}$ \\ Yutao Yan, ${ }^{1}$ Hamed Laroui, ${ }^{1}$ Doron Shmerling, ${ }^{4}$ Tiziano Tallone, ${ }^{4}$ \\ Mauro D'Amato, ${ }^{5}$ Shanthi V. Sitaraman, ${ }^{1}$ and Didier Merlin ${ }^{1,6}$
}

\begin{abstract}
'Department of Medicine, Emory University, Atlanta, Georgia, USA. ${ }^{2}$ Department of Clinical Science Intervention and Technology, Karolinska Institute, Stockholm, Sweden. 3Department of Internal Medicine, Örebro University Hospital, Örebro, Sweden. ${ }^{3}$ PolyGene AG, Rümlang, Switzerland. ${ }^{5}$ Department of Biosciences and Nutrition, Karolinska Institute, Stockholm, Sweden. ${ }^{6}$ Veterans Affairs Medical Center, Decatur, Georgia, USA.
\end{abstract}

\begin{abstract}
Expression of the transmembrane glycoprotein CD98 (encoded by SLC3A2) is increased in intestinal inflammatory conditions, such as inflammatory bowel disease (IBD), and in various carcinomas, yet its pathogenetic role remains unknown. By generating gain- and loss-of-function mouse models with genetically manipulated CD98 expression specifically in intestinal epithelial cells (IECs), we explored the role of CD98 in intestinal homeostasis, inflammation, and colitis-associated tumorigenesis. IEC-specific CD98 overexpression induced gut homeostatic defects and increased inflammatory responses to DSS-induced colitis, promoting colitis-associated tumorigenesis in mice. Further analysis indicated that the ability of IEC-specific CD98 overexpression to induce tumorigenesis was linked to its capacity to induce barrier dysfunction and to stimulate cell proliferation and production of proinflammatory mediators. To validate these results, we constructed mice carrying conditional floxed Slc3a2 alleles and crossed them with Villin-Cre mice such that CD98 was downregulated only in IECs. These mice exhibited attenuated inflammatory responses and resistance to both DSS-induced colitis and colitis-associated tumorigenesis. Together, our data show that intestinal CD98 expression has a crucial role in controlling homeostatic and innate immune responses in the gut. Modulation of CD98 expression in IECs therefore represents a promising therapeutic strategy for the treatment and prevention of inflammatory intestinal diseases, such as IBD and colitis-associated cancer.
\end{abstract}

\section{Introduction}

Colorectal cancer (CRC) is one of the most common types of malignancy (1). A link between chronic inflammation and CRC development has been described, but the contributing factors and the underlying mechanisms are largely unknown $(2,3)$. Ulcerative colitis (UC), a common form of inflammatory bowel disease (IBD), is associated with an increased risk of CRC, and the development of CRC in patients with UC is one of the best clinically characterized examples of association between inflammation and carcinogenesis (4).

CD98 (or CD98 heavy chain; encoded by SLC3A2) is a type II transmembrane protein that covalently links to one of several L-type amino acid transporters (light chains) to form functional heterodimeric large neutral amino acid transport systems $(5,6)$. Following its discovery as a lymphocyte activation antigen (7), CD98 has since been identified in all cell types with the exception of platelets and is expressed at the highest levels in the gastrointestinal (GI) tract and the tubules of the kidney (5). CD98 also associates with integrin $\beta$ subunits, thereby regulating integrin signaling that controls cell proliferation, survival, migration, and epithelial adhesion/polarity (8-10).

CD98 is highly expressed in human melanoma cell lines, regardless of the tissue of origin (11), as well as in several carcinomas, such as squamous cell carcinoma of the larynx (12), lung adenocarcinomas

Authorship note: Hang Thi Thu Nguyen and Guillaume Dalmasso contributed equally to this work.

Conflict of interest: The authors have declared that no conflict of interest exists. Citation for this article: J Clin Invest. 2011;121(5):1733-1747. doi:10.1172/JCI44631.
(13), breast cancer (14), renal cell cancer (where its expression level correlates with the extent of progression, metastasis and malignancy; ref. 15), and various human neoplasms (16). Upregulation of CD98 has also been observed in intestinal inflammation in vitro and in vivo (17-21). Despite the increasing number of studies on this molecule, the potential pathogenetic role of CD98 in CRC, particularly when associated to chronic inflammation, has yet to be explored.

Here we generated 2 genetically manipulated mouse strains that exhibit IEC-specific CD98 overexpression or downregulation and used them as experimental models to investigate CD98's role and mechanism of action in intestinal inflammation and colitis-associated tumorigenesis.

\section{Results}

Analysis of SLC3A2 gene polymorphisms in human IBD. Although increased expression of CD98 in IBD has been reported previously $(18,20,21)$, the mechanism by which CD98 contributes to the pathogenesis of IBD remains unknown. This prompted us to evaluate the potential clinical relevance of genetic variation in SLC3A2 in IBD, with the hypothesis that genetic alterations of CD98 expression or function may contribute to disease susceptibility in humans. To test this hypothesis, we genotyped SLC3A2relevant SNPs in a cohort of 618 Crohn disease (CD) patients, 630 UC patients, and 630 ethnically matched controls and tested them for association with disease. However, no polymorphism in the SLC3A2 gene was significantly associated with IBD susceptibility (Supplemental Figure 1; supplemental material available online with this article; doi:10.1172/JCI44631DS1). 
Characterization of Tg mice with IEC-specific CD98 overexpression. To study the role of CD98 in pathological mechanisms of intestinal inflammation, we sought to genetically manipulate CD98 expression in mice. We produced human CD98-Villin (hCD98-Villin) $\mathrm{Tg}$ mice that overexpress hCD98 specifically in IECs. Tg mice, but not WT mice, exhibited high hCD98 expression in the small intestine, colon, cecum, and (to a much lesser extent) kidney, but not in other organs (Figure 1A). Immunohistochemical analysis verified hCD98 membrane staining of enterocytes in crypts and villi in Tg mice, but not in WT mice (Figure 1B), similar to the previously reported physiological expression of CD98 in mouse enterocytes (22). Tg mice were generally slimmer than their WT littermates, but healthy and with normal phenotype (Figure 1C). Strikingly, about $10 \%$ of $\mathrm{Tg}$ mice showed approximately halved body weight (Figure 1C) and died at early ages (1-4 weeks). These $\mathrm{Tg}$ mice exhibited much higher CD98 mRNA and hCD98 protein expression levels than did their Tg littermates and were therefore classified as Tg-high expression (Tg-HE; Figure 1, D and E). Tg-HE mice developed abnormal GI phenotype, with dramatically longer and larger small intestine and dilated cecum compared with WT and Tg littermates, but no significant difference in size and weight of colons between these groups was observed (Supplemental Figure 2). However, well-formed stool was absent in Tg-HE mice (Supplemental Figure 2), suggestive of diarrhea. Apart from these changes, the morphology of different organs, including the GI tract of most $\mathrm{Tg}$ mice, was apparently normal, as judged by histological examination (Supplemental Figure 3A). A few Tg-HE mice displayed focal chronic colitis with crypt epithelial degeneration and loss associated with neutrophil infiltrates, which was not detected in WT mice (Supplemental Figure 3B). Examination of IEC ultrastructure by electron microscopy showed abnormal morphology and decreased size of microvilli at the apical membranes of enterocytes in Tg compared with WT littermates (Figure 1F). This suggests that IEC-specific CD98 overexpression resulted in a defect of microvilli at the brush border of apical surfaces of mouse villous enterocytes. Because microvilli greatly increase the surface area of the small intestine for digestion/absorption, these data suggest that $\mathrm{Tg}$ mice have a defect in nutrient absorption. Together, these observations suggest a correlation between the levels of hCD98 expression and the abnormality of some phenotype indexes observed in Tg mice.

CD98 Tg mice exhibit barrier dysfunction and dysregulated gene expression profiles. Epithelial barrier function in Tg mice was assessed by measuring the translocation of $4 \mathrm{kDa}$ FITC-dextran, given by gavage, into serum. Compared with WT littermates, enhanced permeability to FITC-dextran was observed in both $\mathrm{Tg}$ groups, with $\mathrm{Tg}$-HE animals displaying a dramatic increase in FITC-dextran translocation into the serum (Figure 2A), indicative of disrupted barrier function.

We then performed a comparative analysis of gene expression profiles in the jejunum and colon of $\mathrm{Tg}$ and $\mathrm{Tg}$-HE versus WT mice, using cDNA microarrays. Of 45,281 transcripts assayed, different signals were detected for 1,039 and 2,548 probe sets, corresponding to 682 and 1,654 known genes in the jejunum of $\mathrm{Tg}$ and $\mathrm{Tg}$-HE mice, respectively, compared with WT animals (Supplemental Table 1, A and B). Although in lower numbers, differentially expressed genes were also detected in the colon: compared with the WT group, Tg and Tg-HE mice generated different signal intensities for 203 and 642 probe sets, representing 125 and 458 known genes, respectively (Supplemental Table 2, A and B).
In general, both the number of genes and the magnitude of their differential expression relative to WT mice were higher in Tg-HE than in $\mathrm{Tg}$ mice, possibly as a result of the respective levels of hCD98 expression. These patterns of differential gene expression were further annotated using the DAVID Bioinformatics Resources 2008 (23), which allows functional categorization of genes into different biological pathways (Supplemental Table 1C and Supplemental Table 2C). Most importantly, expression of the proinflammatory cytokines IL- $1 \beta$ and TNF- $\alpha$ was upregulated in both jejunums and colons of the Tg groups compared with the WT group (Supplemental Table 1C and Supplemental Table 2C), which was also confirmed by quantitative real-time RT-PCR (qRT-PCR) experiments (Figure 2B). Serum levels of TNF- $\alpha$, as quantified by ELISA, were also increased in $\mathrm{Tg}$ and $\mathrm{Tg}$-HE compared with WT mice (Figure 2C). Collectively, these results suggest that IEC-specific CD98 overexpression induces barrier dysfunction and perturbs inflammatory responses, factors both known to contribute to the pathophysiology of IBD.

CD98 Tg mice exhibit increased IEC survival and proliferation. CD98 is known to associate with and regulate integrin signaling, thereby controlling cell growth, survival, and proliferation $(8,9)$. Association of CD98 with $\beta_{1}$-integrin has been previously shown in in vitro model systems (10), and we thus tested whether such interaction could be also detected in mouse IECs. Jejunal and colonic enterocytes from WT mice were extracted, and IP and Western blot (WB) were performed using anti-mouse $\beta_{1}$-integrin (anti- $\mathrm{m} \beta_{1^{-}}$ integrin) and anti-mCD98 antibodies. IP for $\mathrm{m} \beta_{1}$-integrin and WB for mCD98 revealed approximately 80 - to $85-\mathrm{kDa}$ bands corresponding to $\mathrm{CD} 98$, whereas IP for $\mathrm{mCD} 98$ and $\mathrm{WB}$ for $\mathrm{m} \beta_{1}$-integrin revealed a $\beta_{1}$-integrin band of approximately $120-125 \mathrm{kDa}$ in both jejunal and colonic enterocytes (Supplemental Figure 4). We then investigated whether hCD98 expressed in IECs in Tg mice is physiologically associated with $\mathrm{m} \beta_{1}$-integrin. When IECs from WT and $\mathrm{Tg}$ mice were subjected to IP for $\mathrm{m} \beta_{1}$-integrin and WB with anti-hCD98 antibody, which does not recognize mCD98, hCD98 bands were detected in Tg but not WT mice (Figure 3A). Similarly, IP for hCD98 and WB for $\mathrm{m} \beta_{1}$-integrin revealed $\beta_{1}$-integrin bands only in Tg mouse-derived IECs (Figure 3A). These data clearly demonstrate the physiological interaction between hCD98 and $\mathrm{m} \beta_{1}$-integrin in IECs in Tg mice.

Integrin signaling can mediate activation of focal adhesion kinase (FAK), a multifunctional adaptor protein that links integrins to downstream signaling pathways, such as the Ras-Erk and PI3KAkt pathways (24). As shown in Figure 3B, IECs from jejunum of Tg mice exhibited higher levels of phospho-FAK, phospho-Erk1/2, and the proliferation marker cyclin D1 (encoded by Ccnd1). In addition, levels of phospho-Akt and cleaved caspase-3 were respectively increased and decreased in Tg mice (Figure 3B), which suggested an increase in survival of jejunal IECs overexpressing CD98. Levels of phospho-FAK, phospho-Erk1/2, and cyclin D1 were also slightly increased in colonic epithelial cells from $\mathrm{Tg}$ and $\mathrm{Tg}-\mathrm{HE}$ mice compared with WT mice, whereas those of phospho-Akt and cleaved caspase-3 were not substantially changed (Figure 3B). Furthermore, IEC proliferation rate in WT and Tg mice postnatally through the suckling, weaning, and postweaning periods was thoroughly examined. For this purpose, small intestinal and colonic sections from WT and Tg pups by postnatal days 2, 9, 21 (preweaning), and 35 (postweaning) were immunohistochemically stained for the cell proliferation marker Ki67. At all time points, we observed an increase in the number of proliferative intestinal 
A

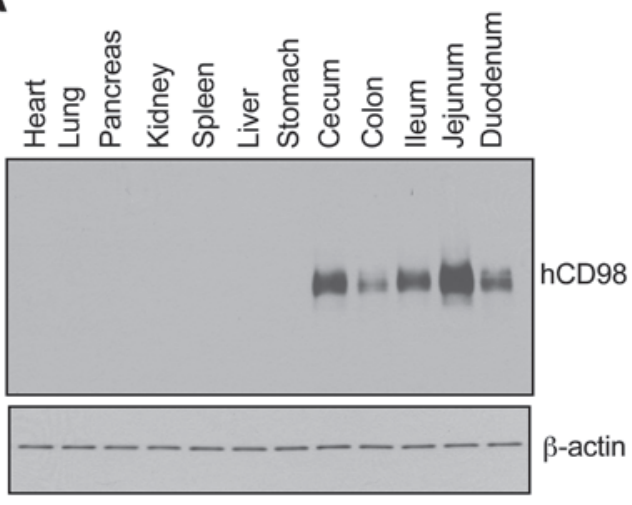

B
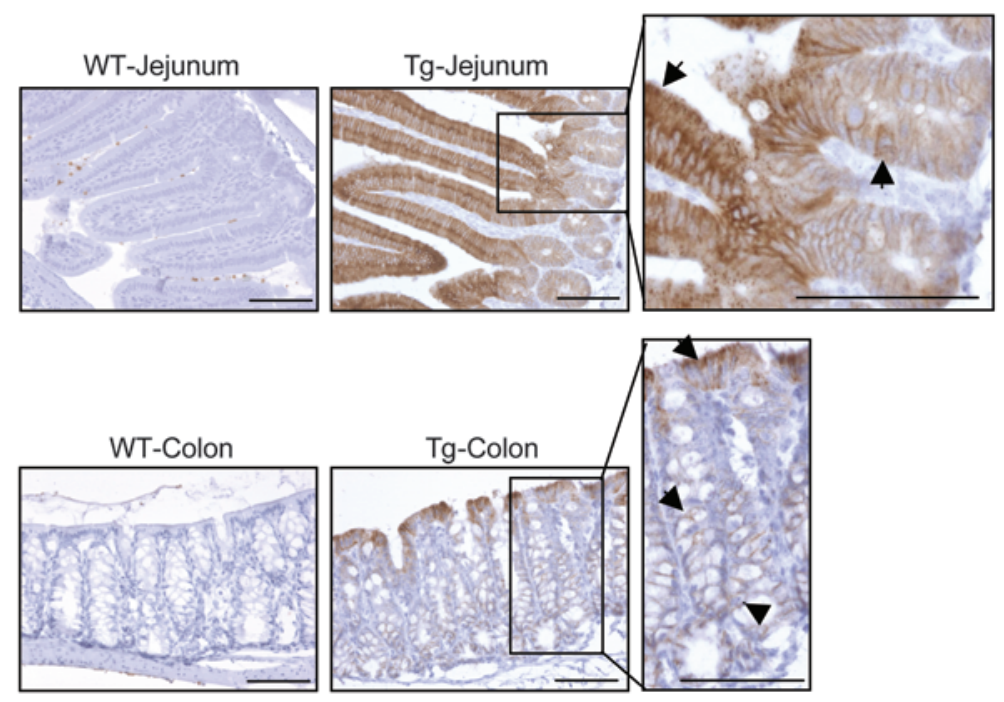

C
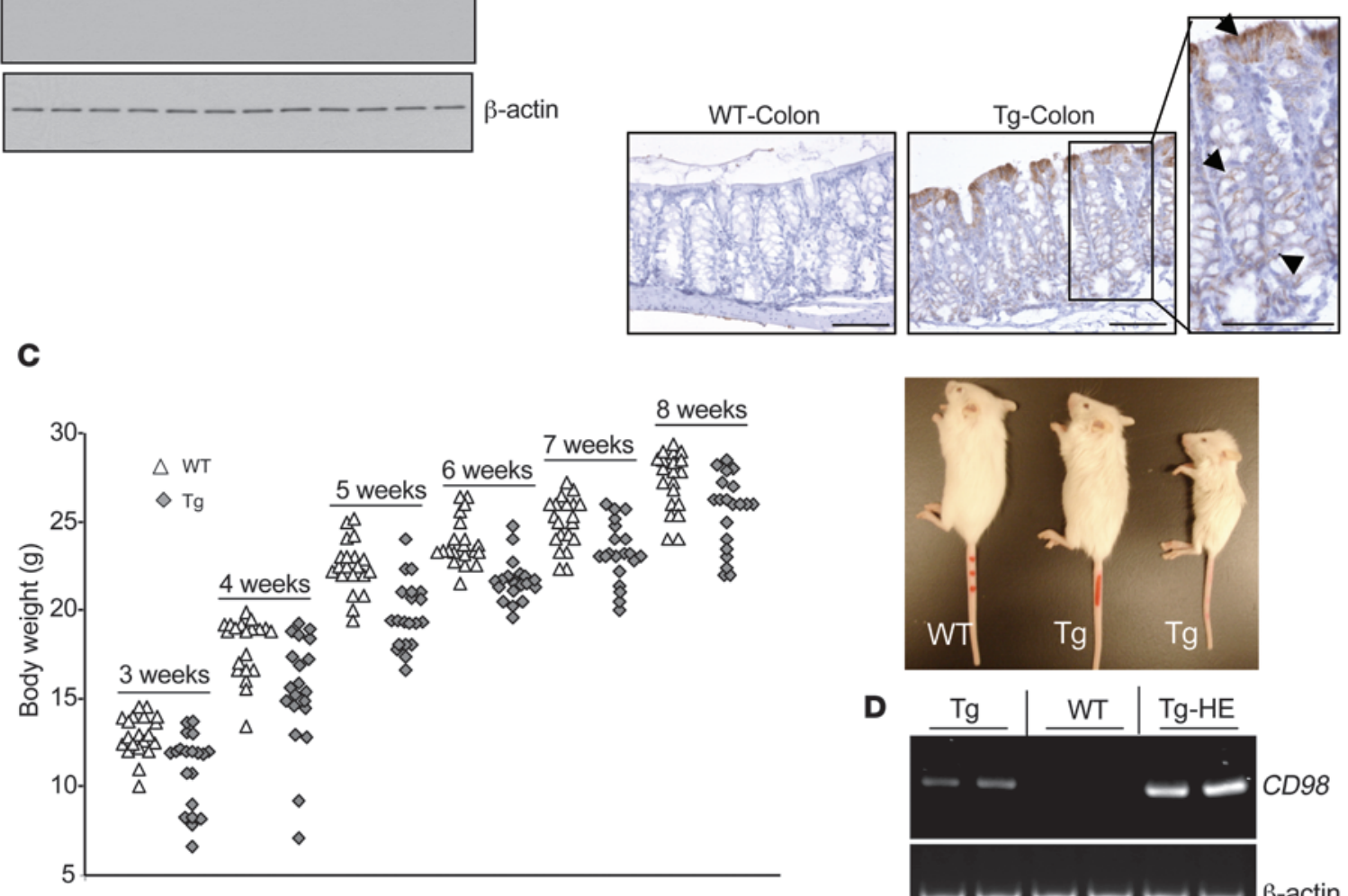

$\mathbf{F}$

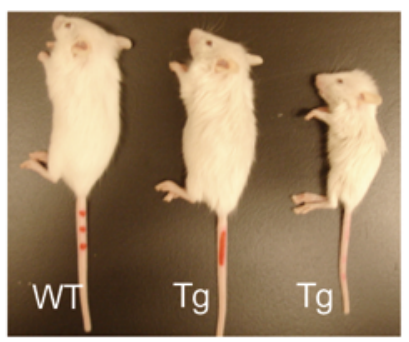

D

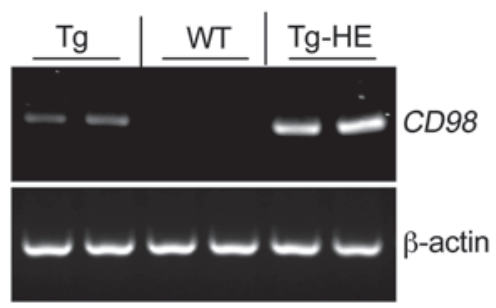

E

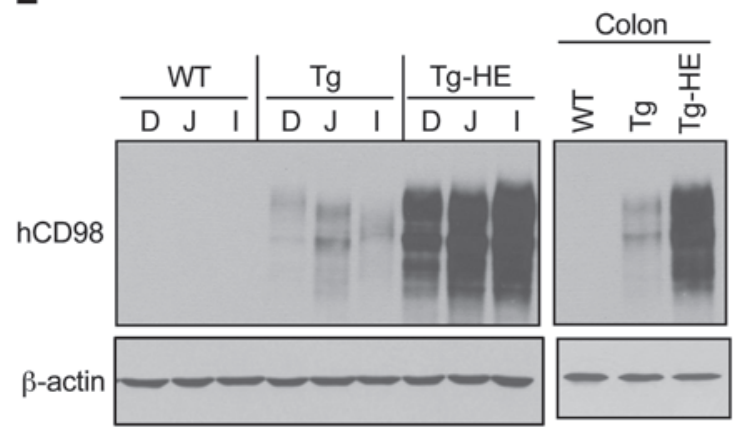

Figure 1

Characterization of Tg mice with IEC-specific CD98 overexpression. IEC-specific hCD98 expression in Tg mice was detected by WB (A) and immunohistochemical analysis (B). The boxed regions are shown enlarged at right. (C) Body weights of age- and sex-matched WT and Tg littermates. (D) CD98 mRNA expression in colonic IECs, analyzed by RT-PCR. (E) hCD98 expression in IECs extracted from mouse duodenum (D), jejunum (J), ileum (I), and colon, assessed by WB. (F) Transmission electron photomicrographs showing abnormal morphology and decreased size of microvilli on the apical membrane of jejunal enterocytes in Tg mice compared with WT littermates. Scale bars: $100 \mu \mathrm{m}$ (B); $1 \mu \mathrm{m}$ (F). 

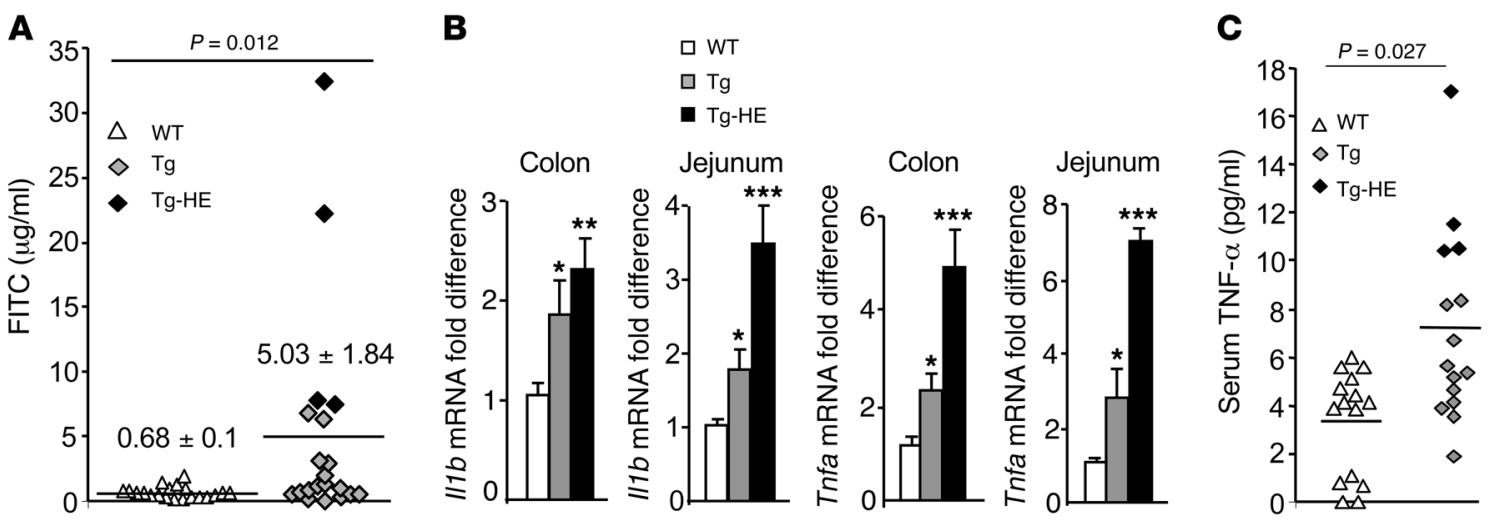

\section{Figure 2}

Tg mice exhibit barrier dysfunction and proinflammatory cytokine upregulation. (A) In vivo permeability assay performed by measuring the translocation of FITC-dextran, given by gavage, into mouse serum after 3 hours. (B) $/ 11 \mathrm{~b}$ and Tnfa levels in jejunum or colonic extracts analyzed by qRT-PCR ( $n=8$ [WT and Tg]; 4 [Tg-HE]). Data are from 1 experiment repeated twice with similar results. ${ }^{*} P<0.05,{ }^{* \star} P<0.005,{ }^{* * *} P<0.001$ vs. WT. (C) Serum levels of TNF- $\alpha$ quantified by ELISA.

crypt cells in Tg compared with WT mice (Figure 3C). Hence, IECspecific CD98 overexpression affects integrin signaling, leading to changes in cell proliferation and survival.

IEC-specific CD98 overexpression increases susceptibility of mice to DSS-induced colitis. Homeostatic defects and epithelial barrier dysfunction, similar to those observed in our $\mathrm{Tg}$ mice, are the hallmarks of IBD (25). We therefore assessed the effect of IEC-specific CD98 overexpression on intestinal inflammation in a dextran sodium sulfate-induced (DSS-induced) colitis model. During the course of DSS treatment, Tg mice displayed more severe body weight loss, rectal bleeding, and diarrhea than did WT littermates, with a higher clinical score determined at day 8 after treatment (Figure 4A). Colon shortening, a macroscopic parameter of colitis severity, was more pronounced in DSS-treated Tg mice than in DSS-treated WT mice (colon length, $4.23 \pm 0.12 \mathrm{~cm}$ vs. $5.21 \pm 0.19 \mathrm{~cm}$; Figure 4B). In addition, DSS-treated Tg mice had a significantly higher histological score, characterized by severe inflammation with loss of entire crypts and surface epithelium in the colon and massive infiltration of inflammatory cells into the mucosa and submucosa (Figure 4C). Activity of myeloperoxidase (MPO), a marker for neutrophil infiltration, in the colon of DSS-treated Tg mice was also markedly increased compared with that in DSS-treated WT mice (Figure 4D). Importantly, as shown in Figure 4E, Tg mice showed a marked increase in DSS-induced colonic production of proinflammatory cytokines (Tnfa, Ifng, Il1b, and Il6) and chemokines (Cxcl1, encoding keratinocyte-derived chemokine; $\mathrm{Cxcl} 2$, encoding macrophage inflammatory protein-2; and $\mathrm{Ccl} 2$, encoding monocyte chemoattractant protein-1). Overall, these results demonstrate that IEC-specific CD98 overexpression aggravates inflammation in a mouse model of colitis.

IEC-specific CD98 overexpression increases colorectal tumorigenesis in a mouse model of colitis-associated cancer. The dramatic effects of IEC-specific CD98 overexpression on intestinal permeability and susceptibility of mice to DSS-induced colitis prompted us to investigate the role of CD98 in inflammation-associated tumorigenesis using a mouse model of colitis-associated cancer (CAC). WT and Tg littermates were intraperitoneally injected with the procarcinogen azoxymethane (AOM) followed by 2 cycles of $2.5 \%$ DSS administration. This treatment caused more weight loss in
Tg than WT mice, and $26.3 \%$ (5 of 19) of the former died versus none of the latter (Figure 5A). Consistent with previous studies, both WT and Tg mice developed tumors in the middle to distal portion of the colon (26), although $\mathrm{Tg}$ mice developed a markedly greater number of tumors (Figure 5B). Average tumor size was also significantly increased in Tg versus WT mice, with tumors larger than $2 \mathrm{~mm}$ in size predominantly found in Tg mice (Figure 5C). Histological examination consistently showed larger adenomas with increased inflammatory cell infiltration in colonic sections from Tg compared with WT mice (Figure 5D). Importantly, during AOM/DSS treatment, induction of colonic MPO activity was increased in Tg compared with WT mice, as was proinflammatory cytokine/chemokine production (Figure 5, E and F), which can promote tumor development (4).

We next assessed IEC proliferation, which can contribute to tumor growth (4), in these mice. Ki67 immunohistochemical staining in untreated animals revealed a slight increase in basal crypt proliferation rate in Tg versus WT mice, whereas AOM/DSS treatment markedly increased the number of Ki67-positive cells in the colonic crypts of Tg mice, in both tumor and nontumor regions, compared with WT mice (Figure 6A). Consistent with this result was a significant increase in Ccnd1 mRNA and cyclin D1 protein levels (Figure 6, B and C). We also examined the IEC proliferation rate during colitis, which can contribute to CAC induction. DSSinduced colonic cyclin D1 protein and Ccnd1 mRNA expression levels were significantly elevated in Tg compared with WT mice (Supplemental Figure 5, A and B). These results indicate that IECspecific CD98 overexpression promotes cell proliferation during both early and late stages of CAC development.

Apoptosis also plays an important role in carcinogenesis, since its suppression can contribute to tumor progression by allowing genetically compromised cells to proliferate (4). The effect of IEC apoptosis on tumor development in WT and Tg mice was assessed by TUNEL assay and WB analysis of cleaved caspase-3 levels. Tg mice exhibited increased DSS-induced apoptosis of colonic epithelium, as shown by their higher TUNEL-positive cell numbers and increased cleaved caspase-3 levels (Supplemental Figure 5, C and D). However, no significant difference in IEC apoptosis was detected between WT and Tg groups after AOM/DSS treatment (Supplemental Figure 6, A and B). Thus, IEC-specific CD98 over- 
A

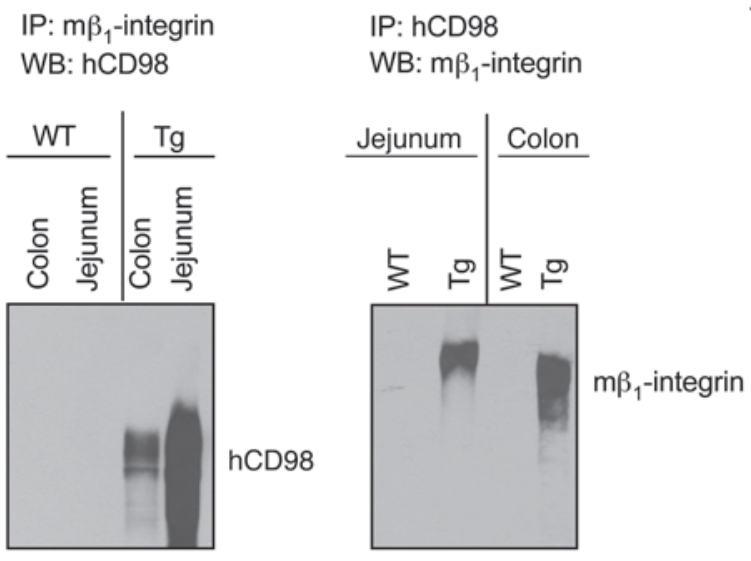

B

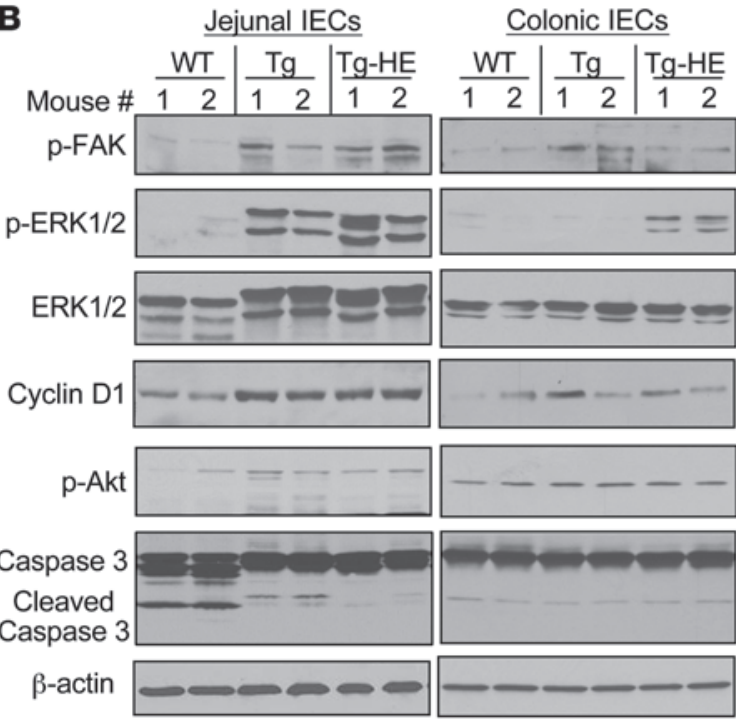

C
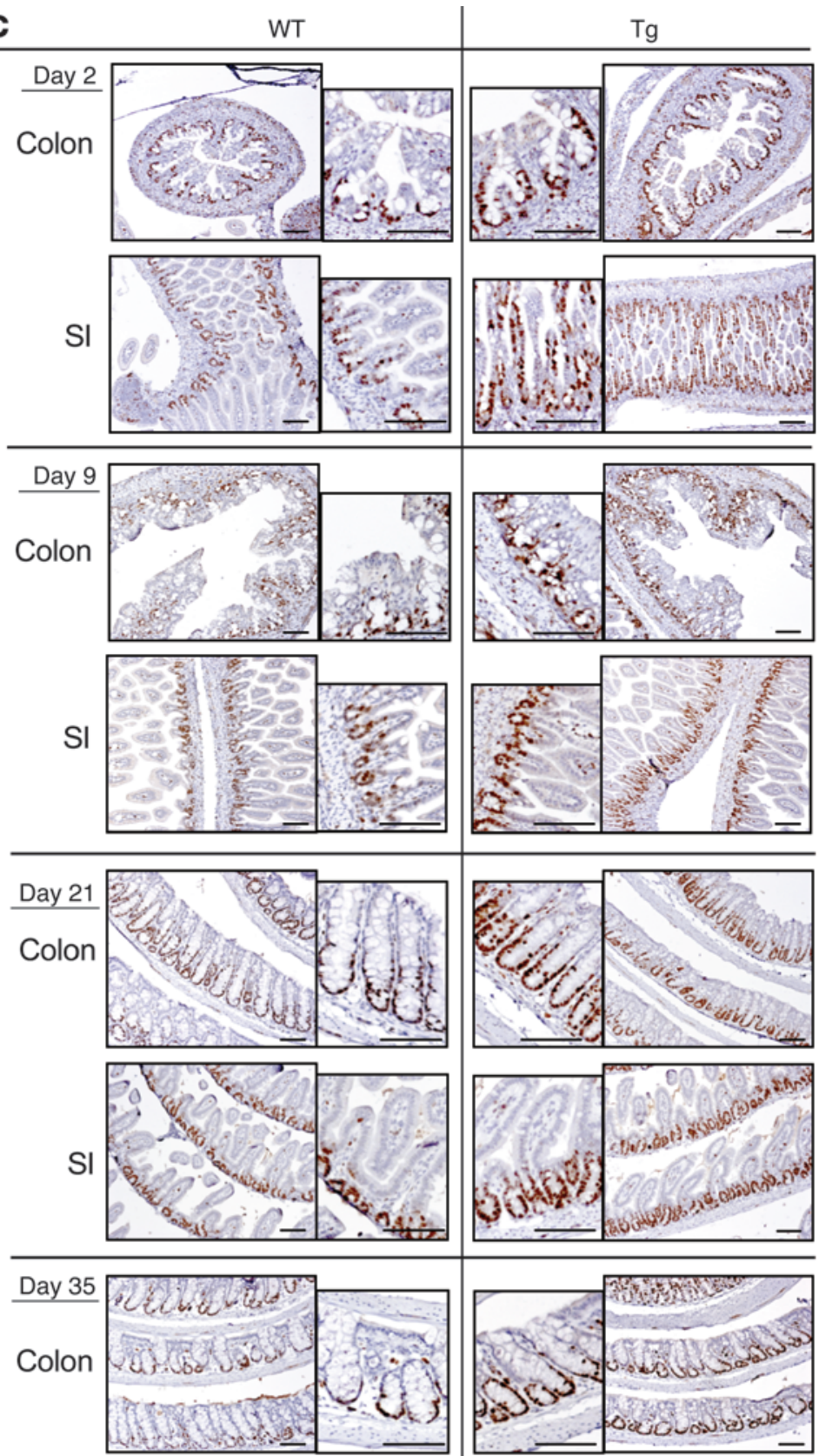

$\mathrm{SI}$
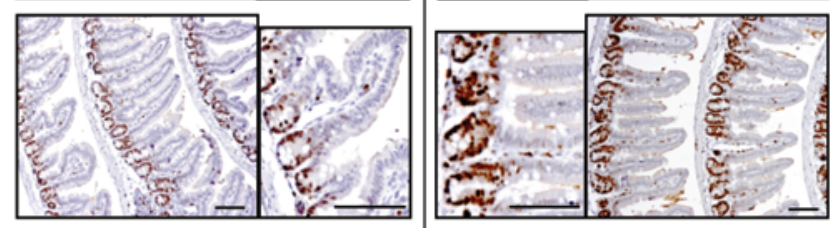
the photomicrographs are enlarged in adjacent panels. Scale bars: $100 \mu \mathrm{m}$.

expression may enhance tumor development by promoting IEC proliferation and proinflammatory cytokine/chemokine production, rather than by decreasing IEC apoptosis.

Chronic inflammation-induced colorectal tumorigenesis in CD98 Tg mice. The contribution of IEC-specific CD98 overexpression to inflammation-induced carcinogenesis was verified in a model of DSS-induced chronic CAC, wherein the use of AOM was omitted. Compared with WT littermates, $\mathrm{Tg}$ mice were more susceptible to long-term, cyclic DSS administration (5 cycles of 2.5\% DSS for 7 days followed by $\mathrm{H}_{2} \mathrm{O}$ for 14 days), as shown by the much higher mortality rate (28\% vs. $0 \%$; Table 1). Most Tg mice showed severe rectal bleeding, diarrhea, and weight loss toward the end of the treatment (data not shown). Remarkably, $83 \%$ of Tg mice surviving after DSS treatment developed $2.2 \pm 0.2$ colonic tumors, whereas no tumor was detected in WT mice (Table 1). Most of the polyps found in Tg mice were 1-2 mm in diameter, with histological characteristics of adenomas (Figure 7 , $\mathrm{A}$ and B). Cell proliferation in the inflamed colon, as examined by Cond1 mRNA levels, was significantly increased in Tg compared 
A
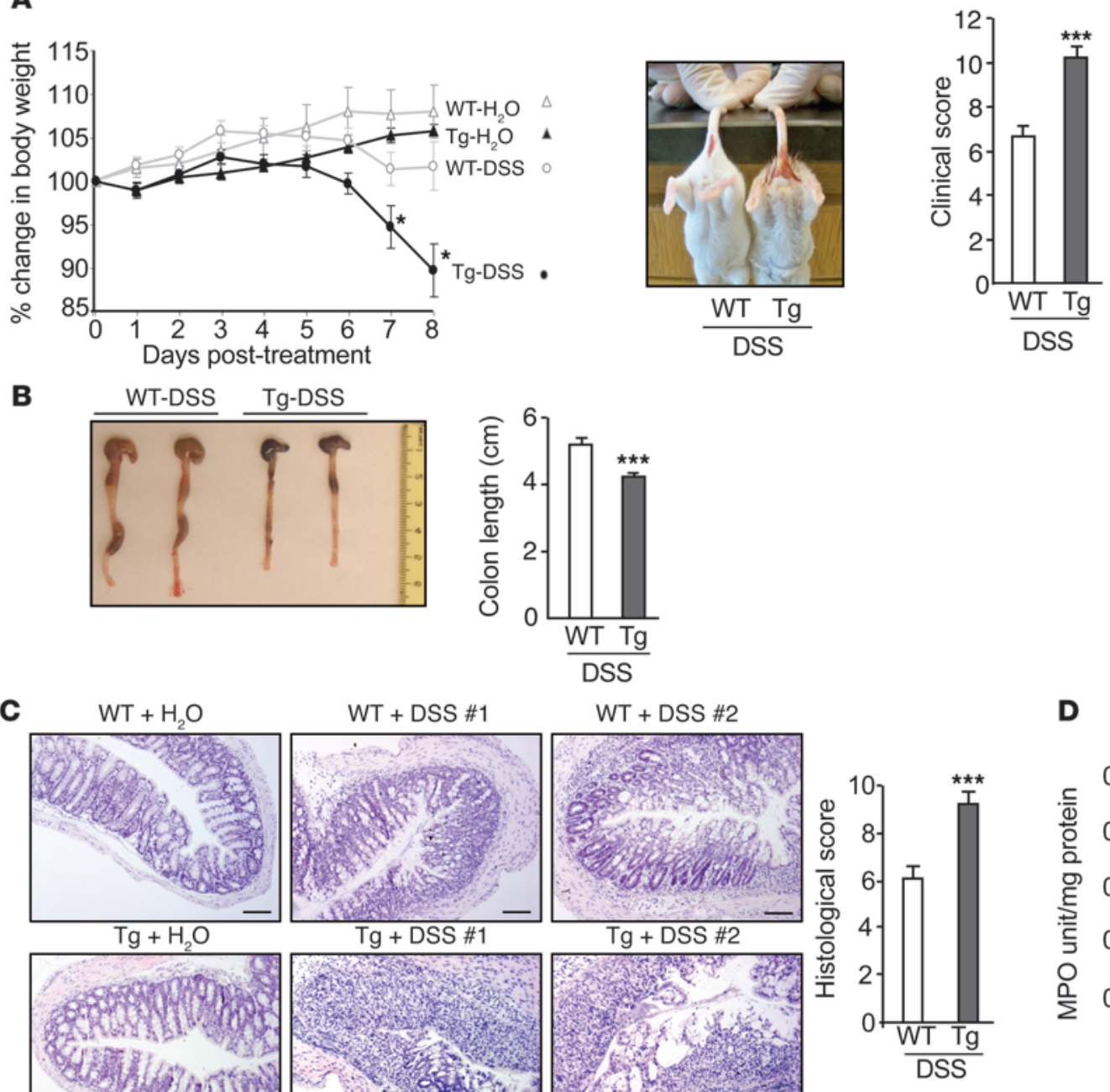

D $\square W T$
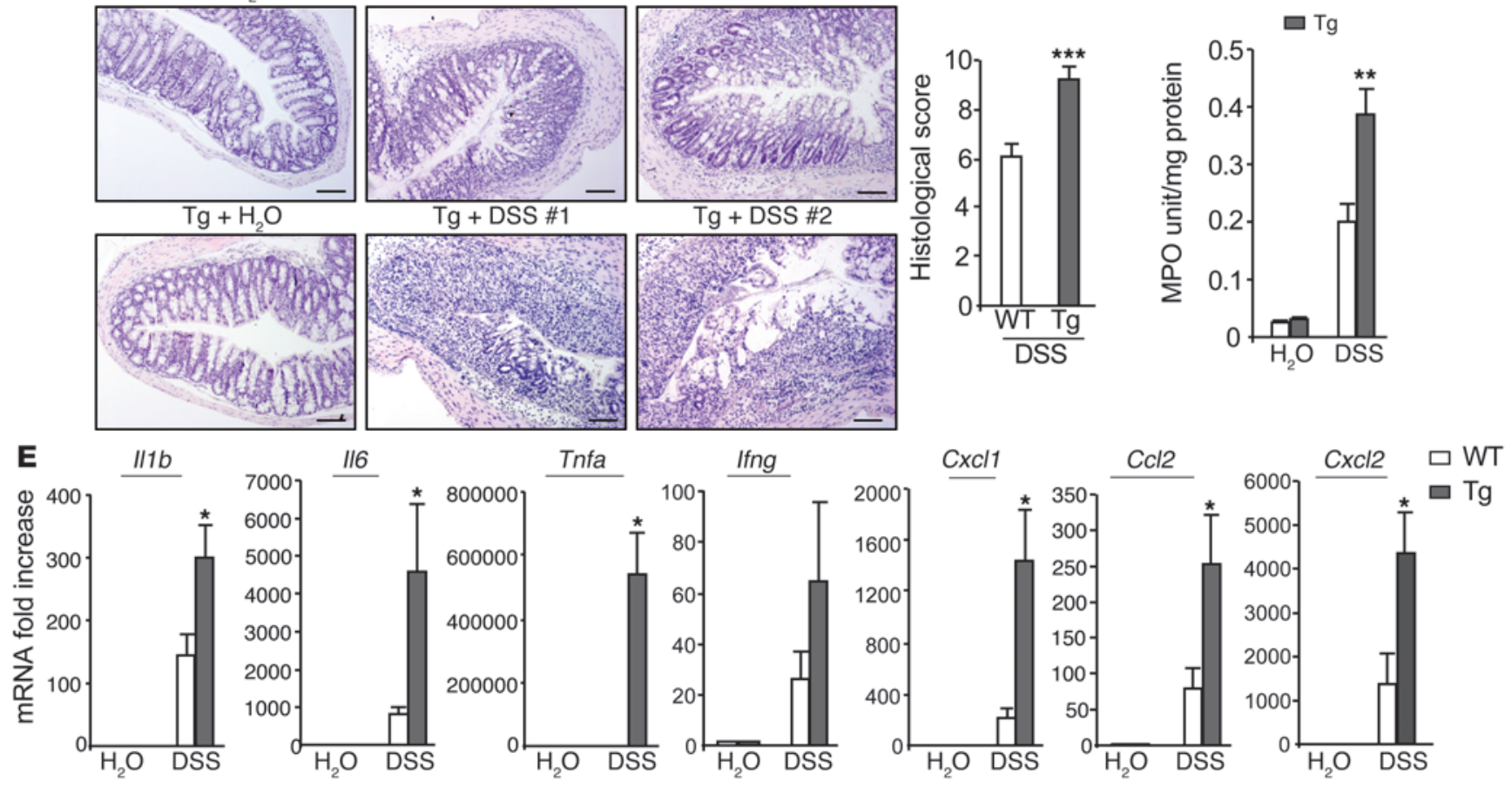

Figure 4

IEC-specific CD98 overexpression increases susceptibility of mice to DSS-induced colitis. WT and Tg littermates were administered regular water (control) or 3.5\% DSS for 8 days. (A) Percent change in body weight was determined during DSS treatment, and clinical score was assessed at the end of the treatment. (B) Colon length, measured at day 8 after treatment. (C) Representative H\&E-stained distal colonic sections and histological score, assessed at day 8 after treatment. Scale bars: $100 \mu \mathrm{m}$. (D) Neutrophil infiltration into the colon, quantified by measuring MPO activity. (E) Colonic cytokine/chemokine mRNA levels, analyzed by qRT-PCR. Data are from 1 experiment repeated twice with similar results ( $n=9$ per group per condition). ${ }^{\star} P<0.05,{ }^{* *} P<0.005,{ }^{* \star *} P<0.001$ vs. WT-DSS.

with WT mice after each DSS cycle, and this was associated with increased induction of proinflammatory cytokines and chemokines (Figure 7, C and D). Together, these results demonstrate that IECspecific CD98 overexpression enhances tumor development and progression by increasing inflammatory responses during chronic colitis, which may represent a causative link among IEC-specific CD98 overexpression, inflammation, and tumorigenesis.

IEC-specific CD98 downregulation decreases susceptibility to DSSinduced colitis and CAC development in mice. The role of CD98 in CAC development was confirmed in mice with IEC-specific CD98 
A
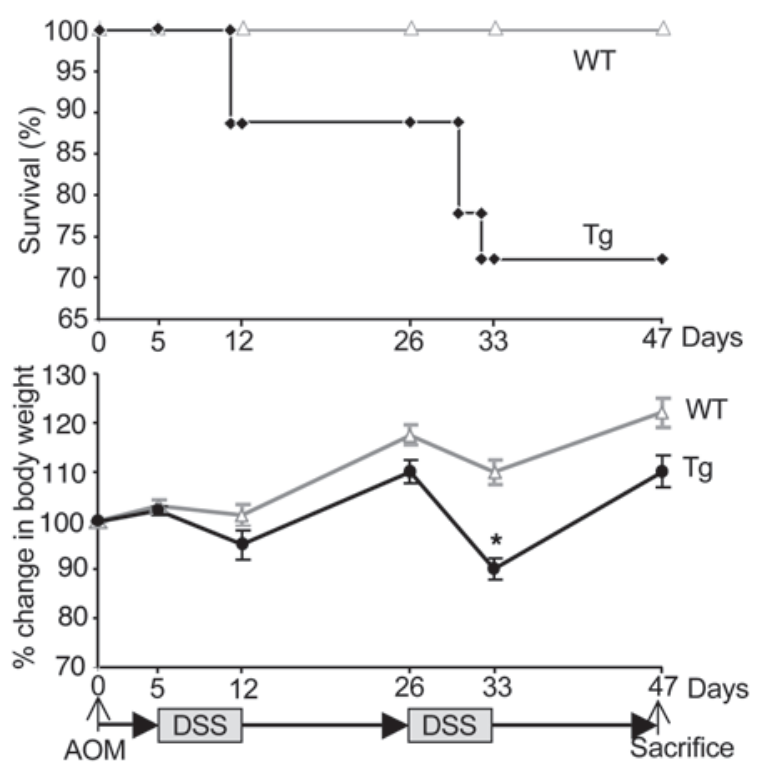

C
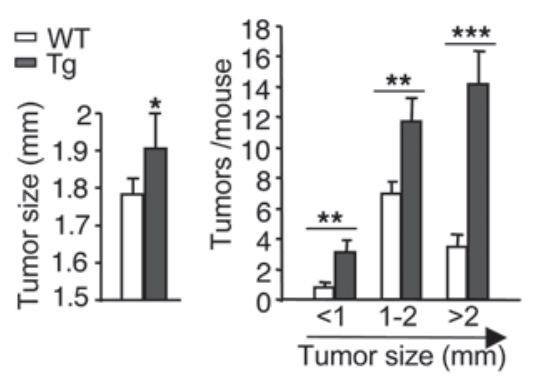

$\mathbf{E}$
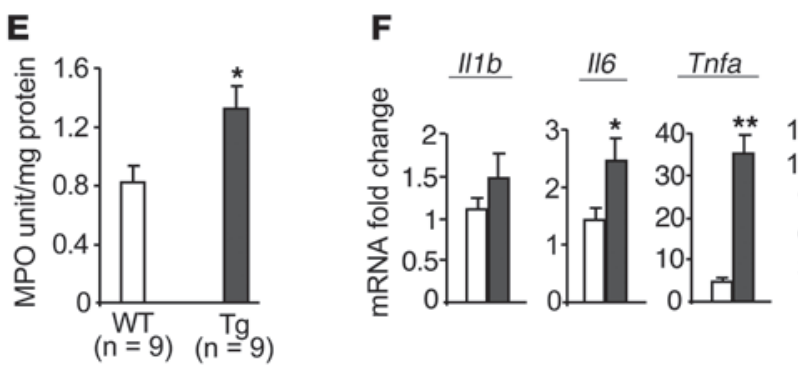

B
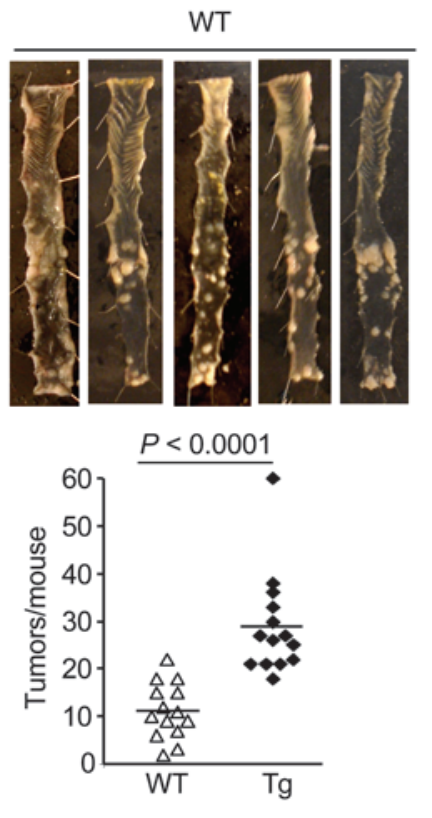
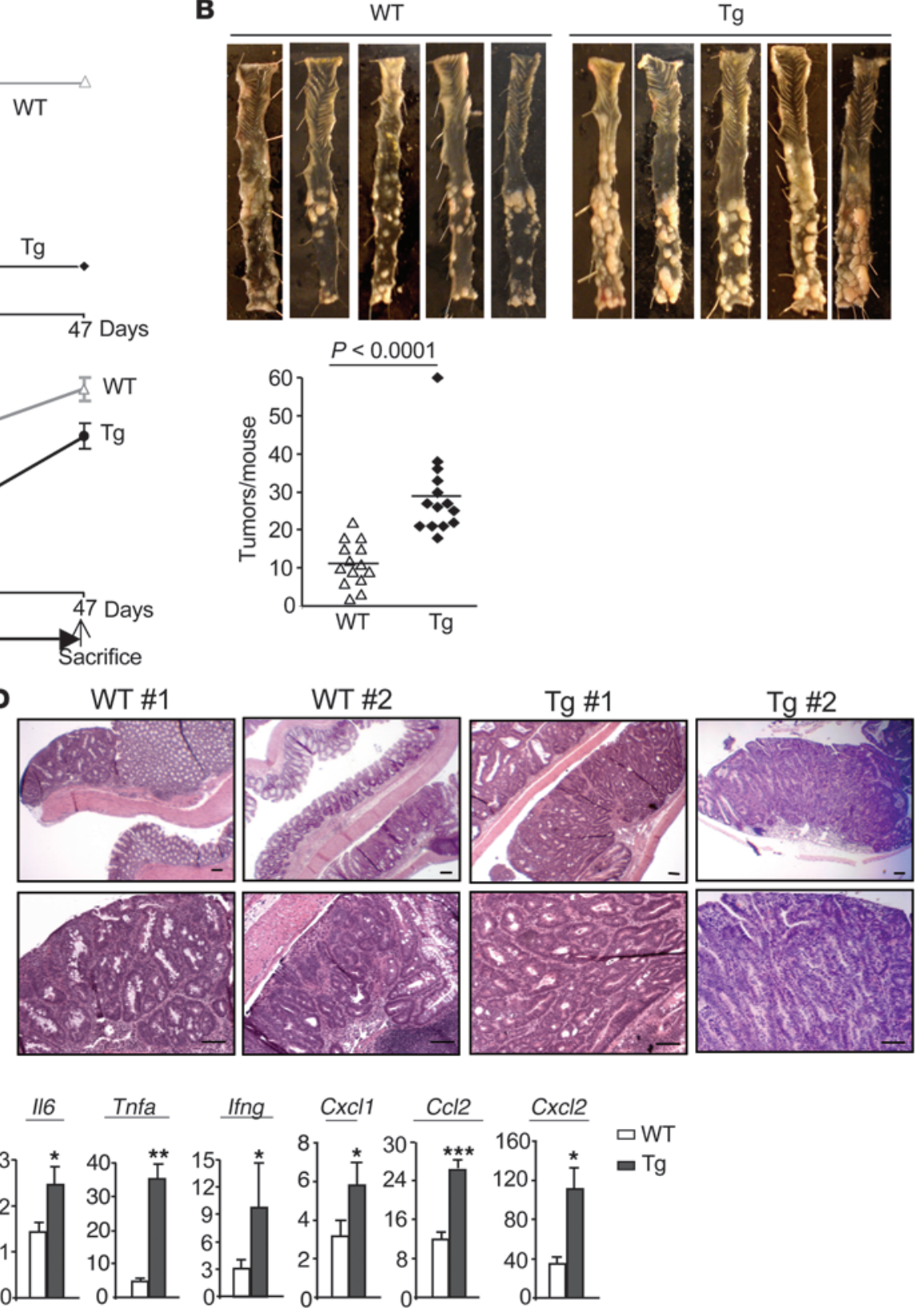

\section{Figure 5}

IEC-specific CD98 overexpression enhances colonic tumorigenesis in a mouse model of CAC. (A) CAC induction protocol. WT and Tg littermates were intraperitoneally injected with AOM $(10 \mathrm{mg} / \mathrm{kg}$ body weight) and maintained for 5 days, then subjected to 2 cycles of DSS treatment ( 1 cycle representing 7 days of $2.5 \%$ DSS followed by 14 days of $\mathrm{H}_{2} \mathrm{O}$ ). Survival (14 of $14 \mathrm{WT} ; 14$ of $19 \mathrm{Tg}$ ) and percent change in body weight during the CAC protocol are shown. (B) Representative colons at the end of the CAC protocol and number of tumors per mouse. (C) Tumor size, determined using a dissecting microscope ( $n=14$ per group). Size distribution of tumors is also shown. (D) Representative H\&E-stained colonic tumors at the end of the CAC protocol. Scale bars: $100 \mu \mathrm{m}$. (E) Colonic MPO activity in AOM/DSS-treated mice. (F) Colonic mRNA levels of cytokines/chemokines in AOM/DSS-treated mice, quantified by qRT-PCR ( $n=9$ per group). Data are from 1 experiment repeated twice with similar results. ${ }^{\star} P<0.05,{ }^{\star \star} P<0.005,{ }^{* \star *} P<0.001$ vs. WT.

downregulation. We generated mice carrying conditional Slc3a2 alleles, with exons 1 and 2 flanked by loxP sites (referred to herein as CD98 $8^{\mathrm{fl} / \mathrm{fl}}$ mice; Supplemental Figure 7), and crossed them with Villin-Cre (VilCre) mice with IEC-specific Cre recombinase expression. The resulting mice (referred to herein as $\mathrm{CD} 98^{\mathrm{fl} /+}$
VilCre) showed Cre-mediated recombination of the floxed Slc3a2 allele in small intestine, colon, and cecum (Figure 8A). CD98 $8^{\mathrm{fl} /+}$ VilCre mice exhibited decreased CD98 protein expression in IECs from small intestine, colon, and cecum compared with control CD98 $8^{\mathrm{fl} /+}$ littermates (Figure 8B). Colonic Cd98 mRNA 
A
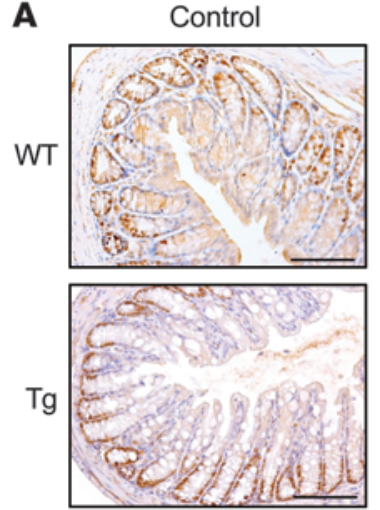

B

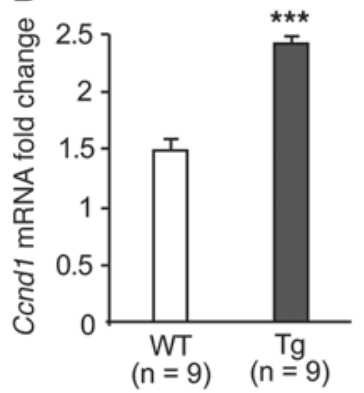

$+\mathrm{AOM} / \mathrm{DSS}$
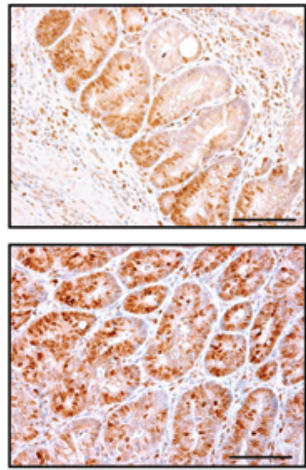

Tumor
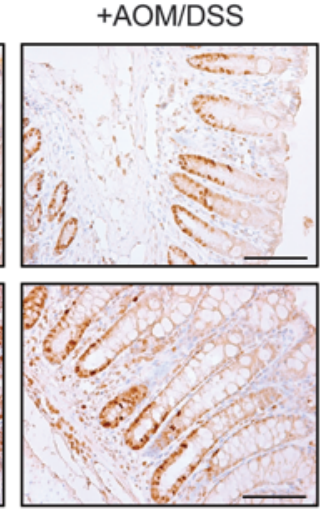

Normal epithelium

C

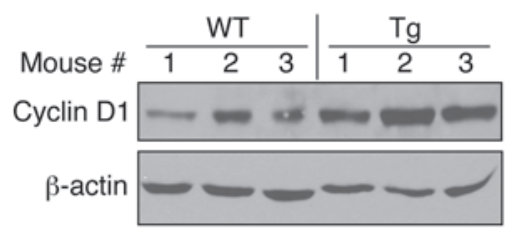

\section{Figure 6}

IEC-specific CD98 overexpression promotes cell proliferation during CAC development. Mice were subjected to the CAC protocol in Figure 5A. (A) Ki67 immunohistochemical staining of colonic sections from untreated (control) and AOM/DSS-treated mice. Scale bars: $100 \mu \mathrm{m}$. Colonic Ccnd1 mRNA and cyclin D1 protein levels in AOM/DSStreated mice were assessed by qRT-PCR $(B)$ and WB $(\mathbf{C})$, respectively. Data are from 1 experiment repeated twice with similar results. ${ }^{\star \star \star} P<0.001$ vs. WT

levels, as analyzed by qRT-PCR, were decreased by $30 \% \pm 5 \%$ in CD $98^{\mathrm{fl} /+}$ VilCre compared with CD98 $8^{\mathrm{fl} /+}$ mice (Figure $8 \mathrm{C}$ ). Despite IEC-specific CD98 downregulation, CD $98^{\mathrm{fl} /+}$ VilCre mice were born at the normal Mendelian ratio, were phenotypically normal, and showed no abnormality in morphology of different organs, including the GI tract, as assessed by histological examination (data not shown). CD98 ${ }^{\mathrm{fl} /+} \mathrm{Vil}$ Cre mice had no evidence of barrier dysfunction, as assessed by measuring serum translocation of 4-kDa FITC-dextran (Figure 8D). These mice also exhibited normal IEC proliferation and survival, as the levels of phospho-FAK, phospho-Erk1/2, cyclin D1, phospho-Akt, and cleaved caspase-3 in IECs were not markedly different from those of CD98 ${ }^{\mathrm{fl} /+}$ mice (Figure 8E). By crossing CD98 $8^{\mathrm{fl} /+}$ VilCre with $\mathrm{CD} 98^{\mathrm{fl} / \mathrm{fl}}$ mice, we obtained CD $98^{\mathrm{fl} / \mathrm{fl}}$ VilCre mice. CD98 $8^{\mathrm{f} / \mathrm{fl}}$ VilCre mice were not born at the normal Mendelian ratio, which indicates that IEC-specific CD98 ablation is associated with embryonic or neonatal lethality. Therefore, $\mathrm{CD} 98^{\mathrm{fl} /+} \mathrm{VilCre}$ mice were used to assess the effect of IEC-specific CD98 downregulation on colitis and CAC development.

Compared with CD98 $8^{\mathrm{fl} /+}$ mice, $\mathrm{CD} 98^{\mathrm{fl} /+}$ VilCre mice were more resistant to 3\% DSS treatment, as shown by reduced body weight loss, decreased rectal bleeding, and no diarrhea, associated with a significantly lower clinical score assigned on day 9 after treatment (Figure 9A). Colon shortening was also reduced in DSStreated CD $98^{\mathrm{fl} /+}$ VilCre compared with DSS-treated CD $98^{\mathrm{f} /+}$ mice
(Figure 9B). Histological scores correlated well with clinical scores, showing attenuated colitis severity in DSS-treated CD98 $8^{\mathrm{fl} /+} \mathrm{VilCre}$ mice, with markedly reduced colonic crypt damage and fewer inflammatory infiltrates and ulcerations compared with DSS-treated CD98 ${ }^{\mathrm{fl} /+}$ mice (Figure 9C). Importantly, DSS-induced colonic mRNA production of proinflammatory cytokines and chemokines was significantly lower in CD98 ${ }^{\mathrm{fl} /+}$ VilCre than in $\mathrm{CD} 98^{\mathrm{fl} /+}$ mice (Figure 9D). These results demonstrate that IEC-specific CD98 downregulation induces resistance to DSS-induced colitis.

As expected, IEC-specific CD98 downregulation decreased CAC development in mice. CD $98^{\mathrm{fl} /+}$ VilCre mice were less susceptible to AOM/DSS treatment than were $\mathrm{CD} 98^{\mathrm{fl} /+}$ mice, as shown by reduced body weight loss and lower mortality rate (Figure 10A). Colonic tumors were lower both in numbers and in size in $\mathrm{CD} 98^{\mathrm{fl} /+} \mathrm{Vil}$ Cre compared with $\mathrm{CD} 98^{\mathrm{fl} /+}$ mice (Figure 10, B and C), and histological examination consistently showed smaller adenomas with decreased colonic inflammatory cell infiltration after exposure to the CAC protocol (Figure 10D). Notably, CD98 $8^{\mathrm{fl} /+}$ VilCre mice exhibited decreased AOM/DSS-induced production of proinflammatory cytokines and chemokines (Figure 10E) and attenuated proliferation rates of colonic epithelial cells, as shown by reduced Ki67-positive cell numbers and cyclin D1 expression levels (Figure 11, A-C). The proliferation rate of colonic epithelial cells during colitis was also decreased in $\mathrm{CD} 98^{\mathrm{fl} /+}$ VilCre mice compared with CD98 $8^{\mathrm{fl} /+}$ mice (Supplemental Figure 8, A and B). No significant difference in IEC apoptosis between $\mathrm{CD} 98^{\mathrm{fl} /+} \mathrm{VilCre}$ and $\mathrm{CD} 98^{\mathrm{fl} /+}$ mice in response to DSS (Supplemental Figure 8, C and D) or AOM/DSS treatment (Supplemental Figure 9, A and B) was observed. These results collectively demonstrated that IEC-specific CD98 downregulation attenuates tumor incidence and tumor size in a mouse model of CAC by decreasing inflammation-induced cytokine/chemokine production and IEC proliferation.

\section{Discussion}

To our knowledge, the role of CD98 in colorectal carcinogenesis has not previously been investigated, at least in part because of the lack of experimental models with modified CD98 expression and/or

\section{Table 1}

Chronic colitis-induced colonic tumorigenesis in $\mathrm{Tg}$ mice

\begin{tabular}{lcc}
\hline & WT & Tg \\
Dead mice & 0 of $25(0 \%)$ & 7 of $25(28 \%)$ \\
Tumor incidence & 0 of $25(0 \%)$ & 15 of $18(83 \%)$ \\
Tumor multiplicity & - & $2.2 \pm 0.22$ \\
Tumor size $(\mathrm{mm})$ & - & $1.65 \pm 0.06$
\end{tabular}

WT and Tg littermates ( $n=25$ per group) were subjected to 5 cycles of DSS treatment ( 7 days of $2.5 \%$ DSS plus 14 days of $\mathrm{H}_{2} \mathrm{O}$ per cycle). Surviving mice were sacrificed after 5 cycles, and colonic tumors were counted and measured using a dissecting microscope. Values are from 1 experiment. AExpressed as no. tumors/mouse. 
A

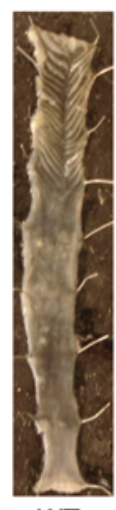

WT
B

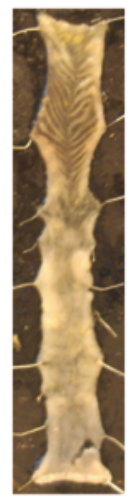

WT
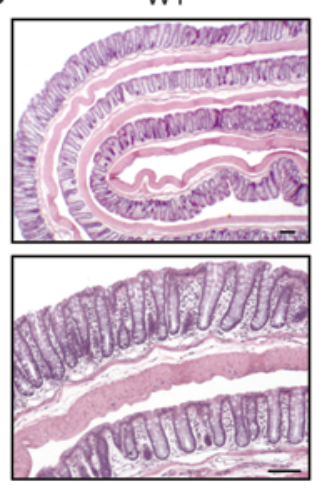

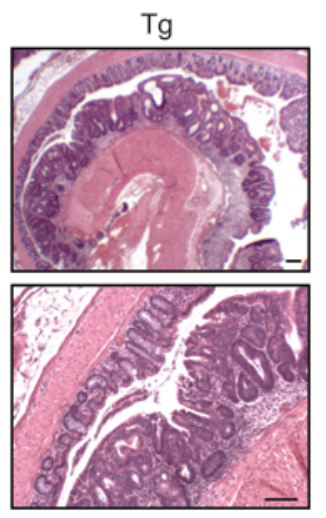

C

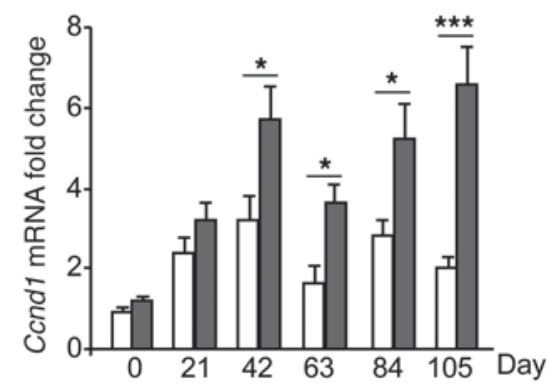

D
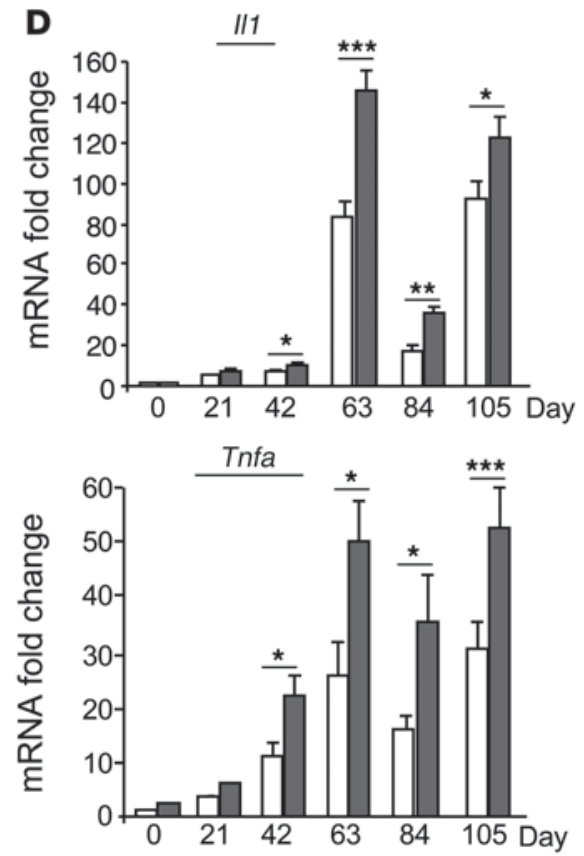
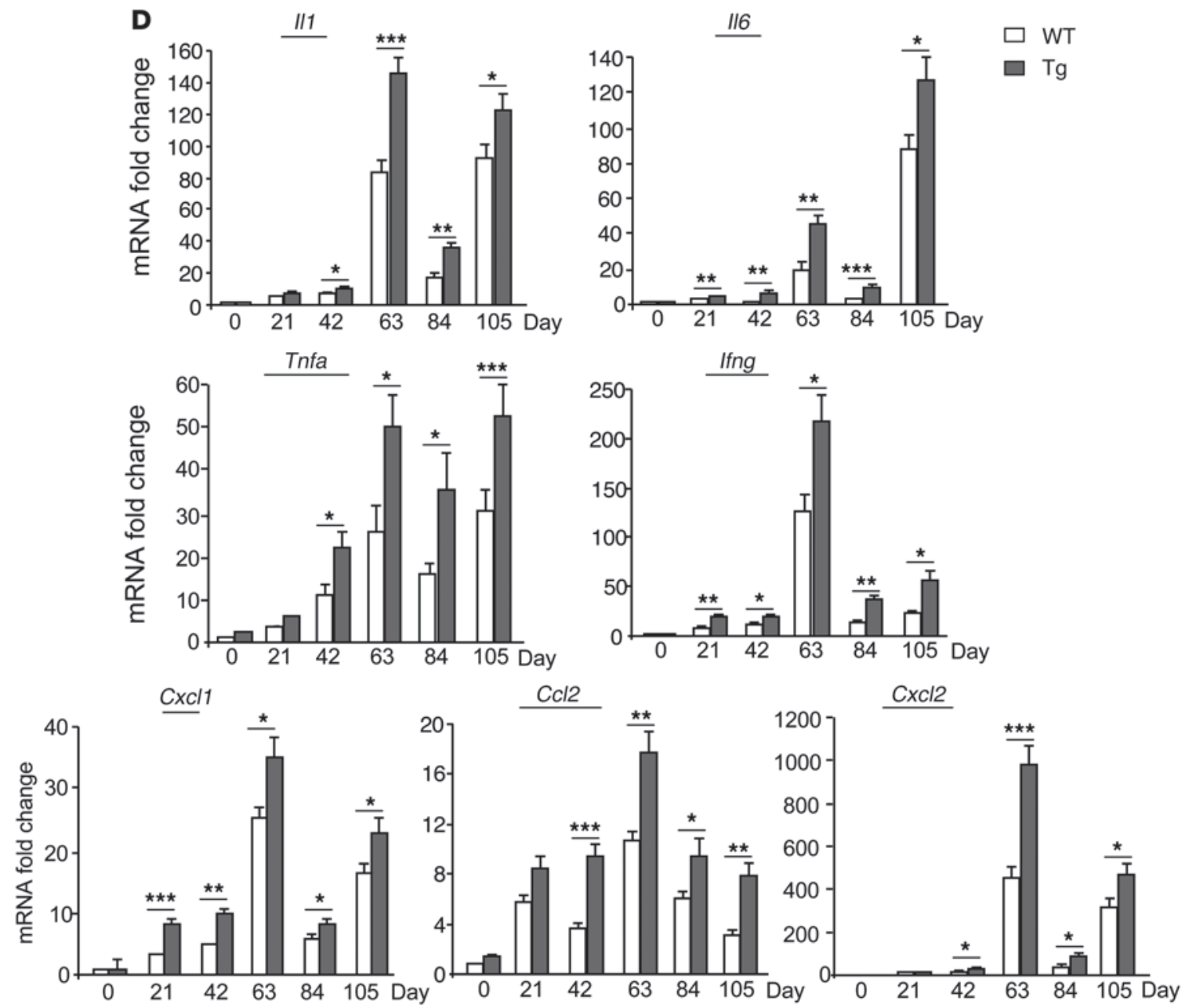

Figure 7

Chronic colitis-induced colonic tumorigenesis in Tg mice. WT and Tg littermates were subjected to 5 cycles of DSS treatment (1 cycle representing 7 days of $2.5 \%$ DSS followed by 14 days of $\mathrm{H}_{2} \mathrm{O}$ ). Representative colons $(\mathbf{A})$ and $\mathrm{H}$ \&E-stained colonic tumors $(\mathbf{B})$ at the end of the cyclic DSS treatment. Scale bars: $100 \mu \mathrm{m}$. Mice were sacrificed after each cycle of the treatment, and colonic mRNA levels of Ccnd1 (C) and cytokines/chemokines (D) were quantified by qRT-PCR. Data are from 1 experiment $\left(n=6\right.$ per group). ${ }^{*} P<0.05,{ }^{* *} P<0.005,{ }^{* * *} P<0.001$ vs. WT.

function. Here we generated 2 mouse models in which CD98 expression was genetically manipulated specifically in IECs and explored the role of CD98 and its mechanism of action in intestinal inflammation and inflammation-associated tumorigenesis.
It is widely recognized that inflammation can contribute to tumor formation and growth (3) and that the strongest association of chronic inflammation with malignant diseases is in colon carcinogenesis arising in IBD patients (2). Here we showed that IEC-specific CD98 
A
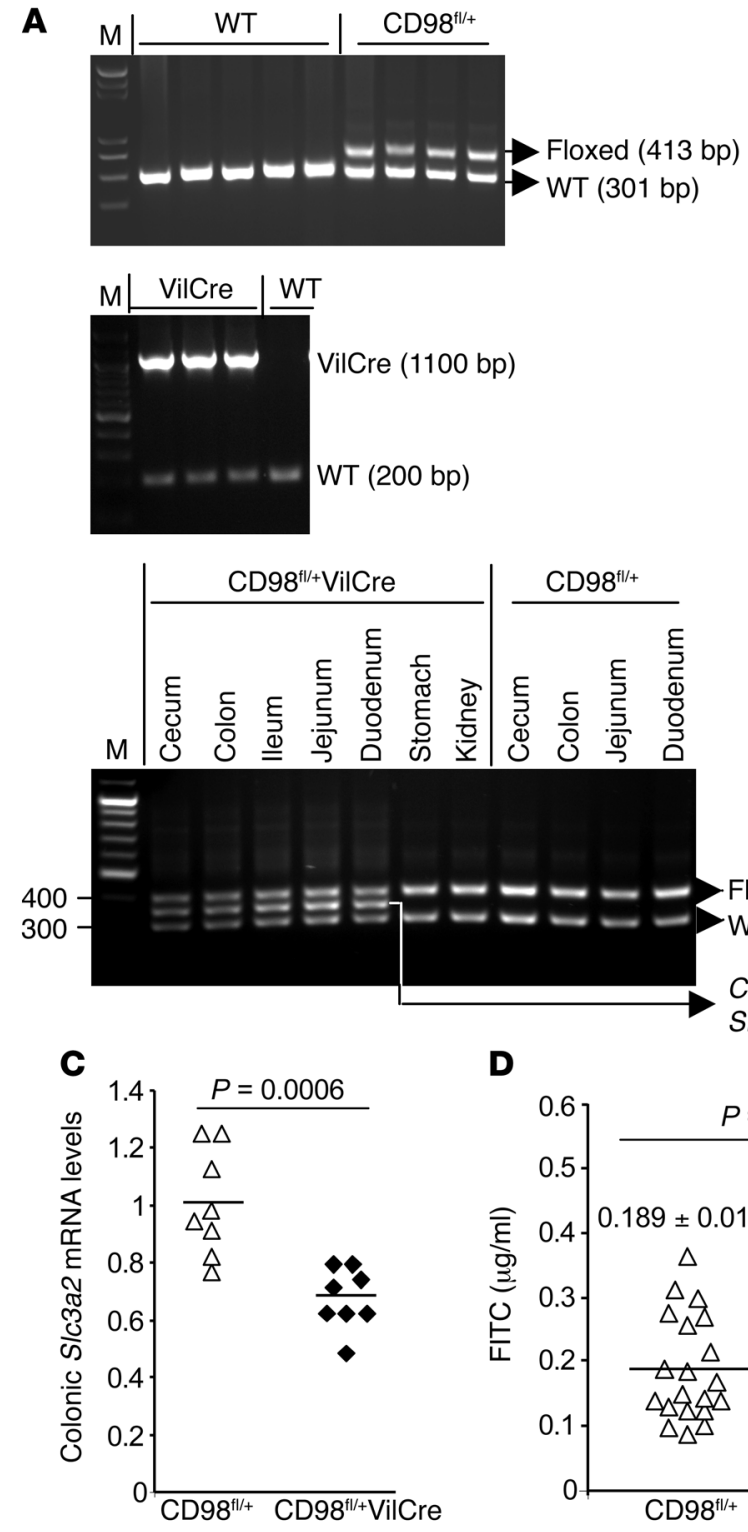

B
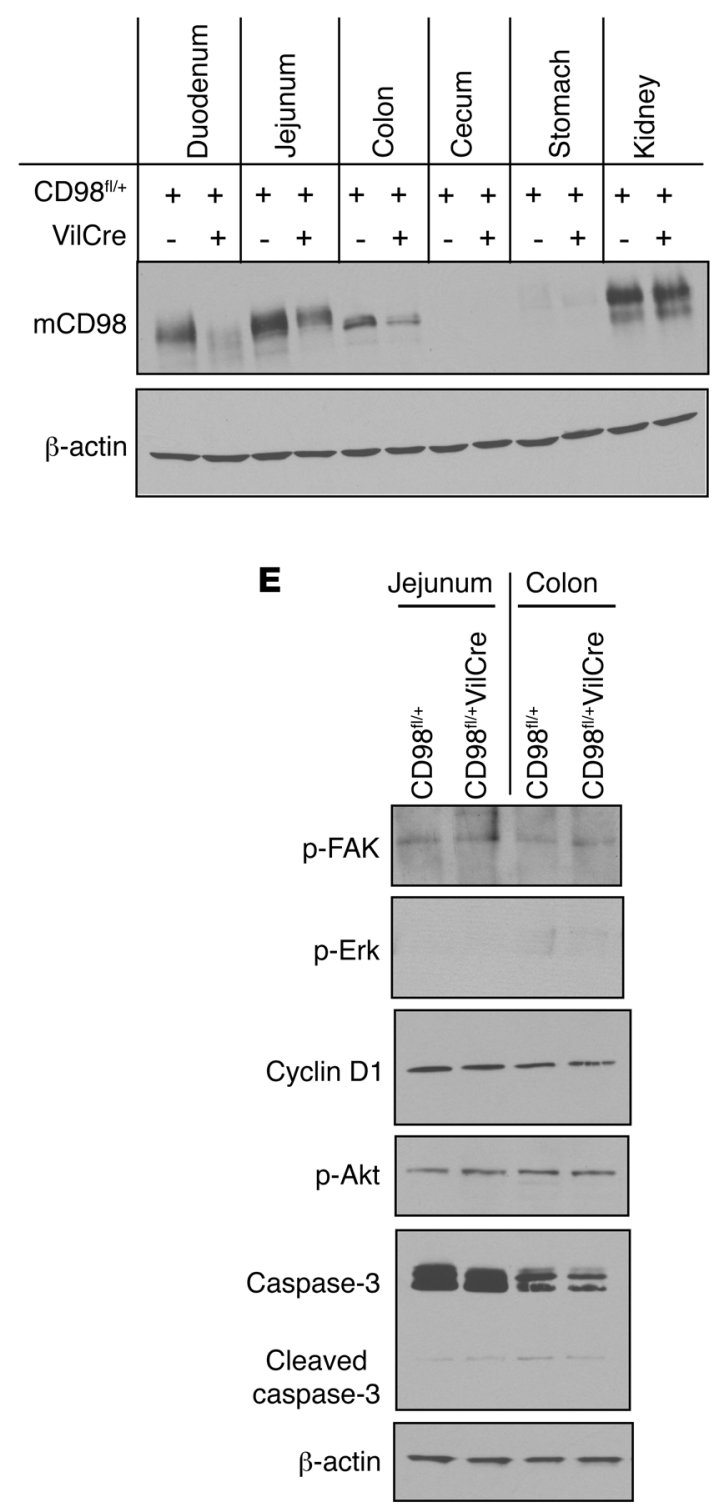

Figure 8

Conditional deletion of CD98 in mouse intestinal epithelium. (A) Verification of the presence of the floxed S/c3a2 allele or VilCre Tg by PCR of tail genomic DNA. PCR products of genomic DNA extracted from different tissues are also shown; the 356-bp product indicates Cre-mediated recombination of the floxed S/c3a2 allele. M, 100-bp DNA ladder (BioLabs). (B) mCD98 expression in different tissues, analyzed by WB. (C) S/c3a2 mRNA levels in colonic IECs, analyzed by qRT-PCR. (D) In vivo permeability assay, performed by measuring the translocation of FITCdextran (given by gavage) into mouse serum after 3 hours. (E) Expression of cell proliferation and apoptosis markers in IECs analyzed by WB.

overexpression promoted colonic tumorigenesis in mouse models of CAC by aggravating intestinal inflammation, with increased IEC proliferation and induction of proinflammatory cytokines and chemokines crucially contributing to tumor progression (2). Our previous studies demonstrated upregulation of CD98 expression in cultured IECs stimulated with proinflammatory cytokines $(19,21)$, in colonic tissues from mice with active colitis (17), and in colonic biopsies from IBD patients (21). We now provide direct evidence that IEC-specific CD98 overexpression aggravates intestinal inflammation in a mouse model of DSS-induced colitis, accompanied by massively increased production of proinflammatory cytokines and chemokines. Collectively, these results prompted us to explore the possibility that alterations in CD98 function and/or expression may contribute to human IBD, but our analysis of SLC $3 A 2$ polymorphisms in CD and UC patients did not disclose any significant finding supporting this hypothesis. However, diverse plausible mechanisms underlying the contribution of CD98 to intestinal inflammation can be envisaged, unrelated to specific genetic predisposing backgrounds. One is that IEC-specific CD98 overexpression results in intestinal barrier dysfunction, among the earliest events that precede evident inflammation or mucosal damage (25) and spontaneous upregulation of proinflammatory genes. Another possible mechanism is that CD98 overexpression affects integrin signaling in IECs, disrupting the homeostatic regulation of cell proliferation and survival, which 
A
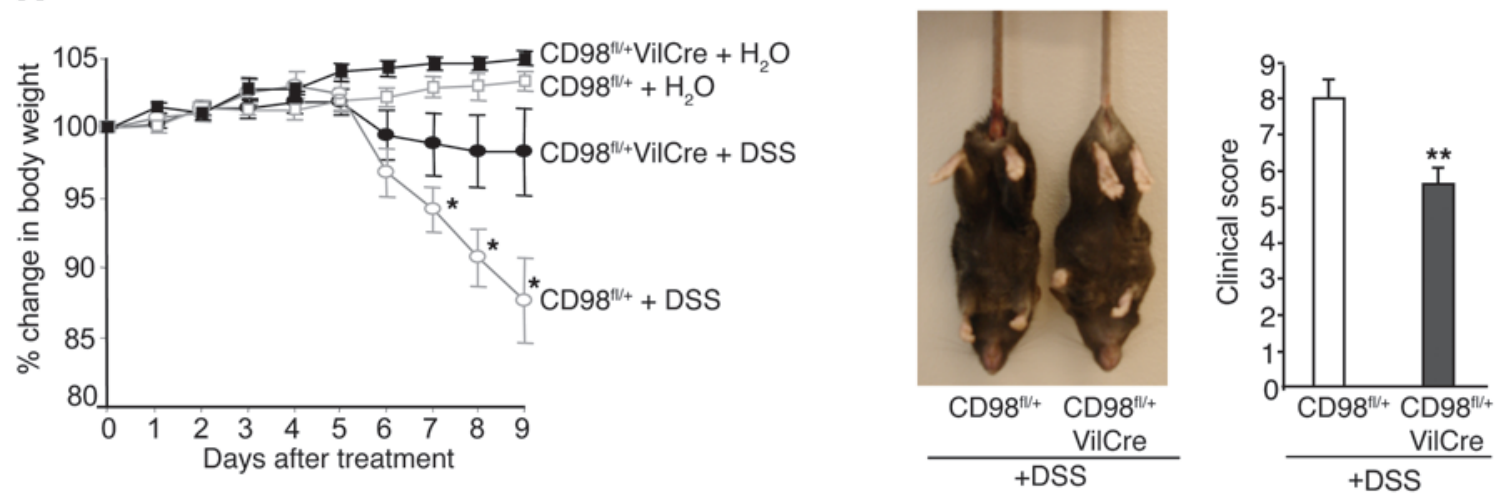

B +DSS

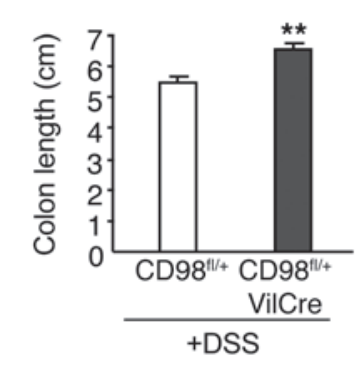

CD98 ${ }^{11+}+\mathrm{Vil}$ Cre

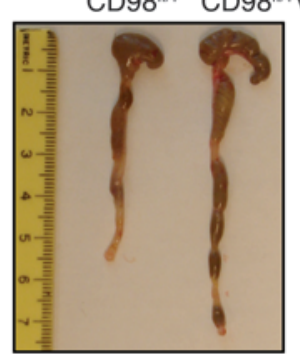

C
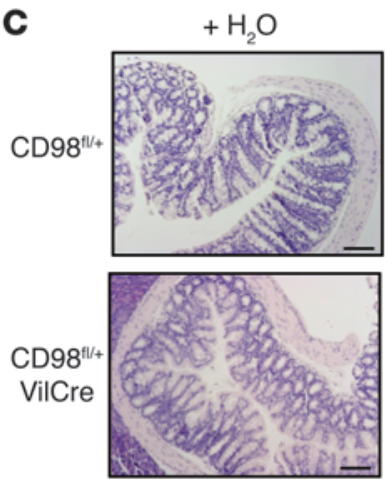

+ DSS \#1
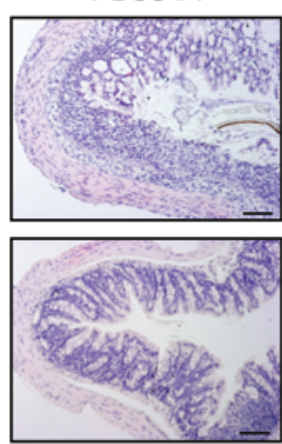

+ DSS \#2
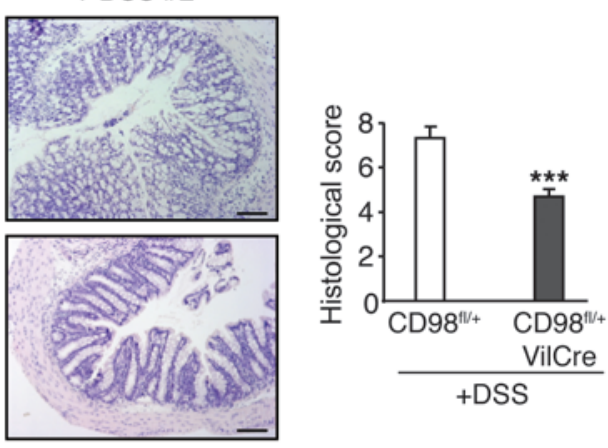

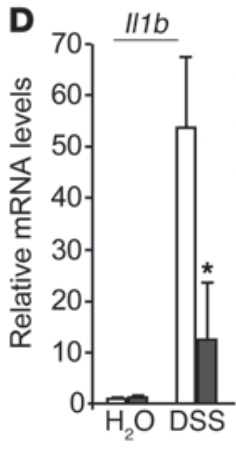
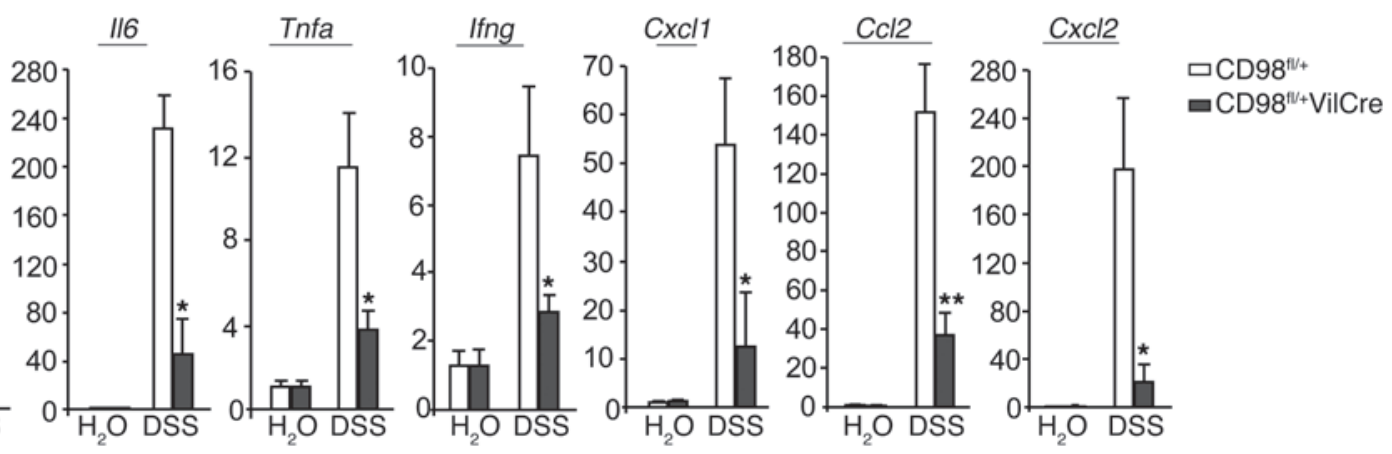

Figure 9

IEC-specific CD98 downregulation protects mice from DSS-induced colitis. Mice were administered regular water (control) or $3 \%$ DSS for 9 days. (A) Percent change in body weight, measured during DSS treatment, and clinical score, assessed at the end of the treatment. (B) Colon length, measured at day 9 after treatment. (C) Representative H\&E-stained distal colonic sections and histological score, assessed at the end of DSS treatment. Scale bars: $100 \mathrm{\mu m}$. (D) Colonic cytokine/chemokine mRNA levels, analyzed by qRT-PCR. Data are from 1 experiment repeated twice with similar results $\left(n=9\right.$ per group per condition). ${ }^{\star} P<0.05,{ }^{* \star} P<0.005,{ }^{* \star *} P<0.001$ vs. DSS-treated CD $98^{\mathrm{fl} /+}$. 
A
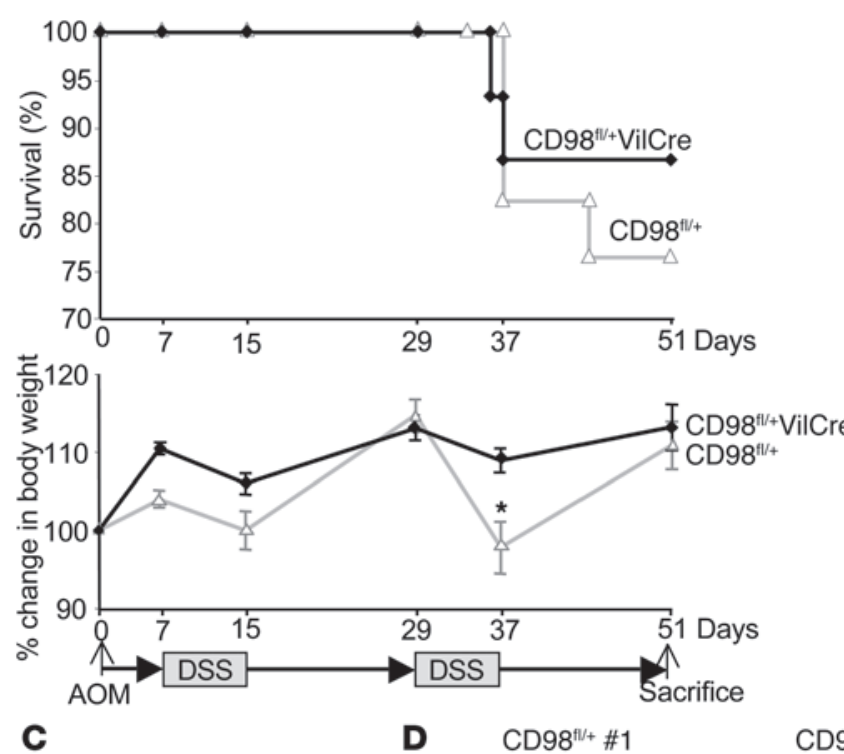

c

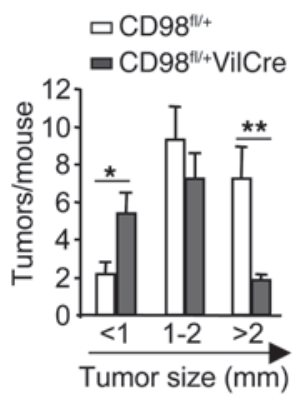

B
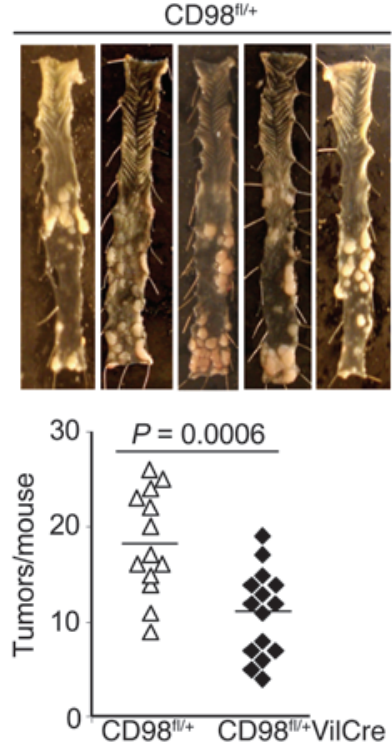

D9 $98^{\mathrm{fl} / \mathrm{+}}$ \#2

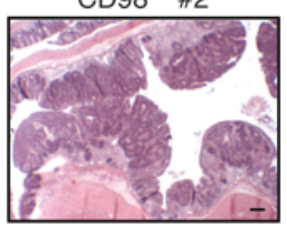

$\mathrm{CD}^{\mathrm{ft} /+}+\mathrm{VilCre} \# 1$

$\mathrm{CD}^{\mathrm{ft} /}+\mathrm{Vil}$ re \#2
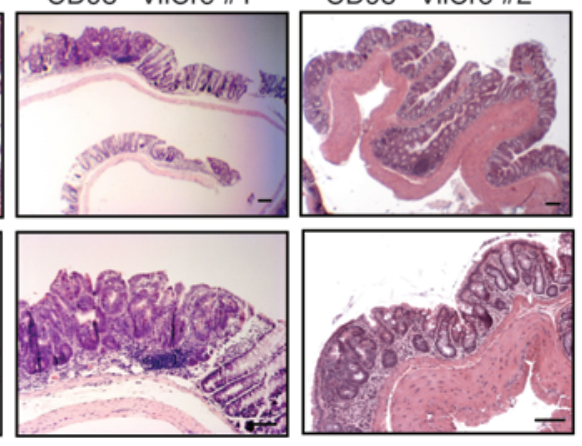
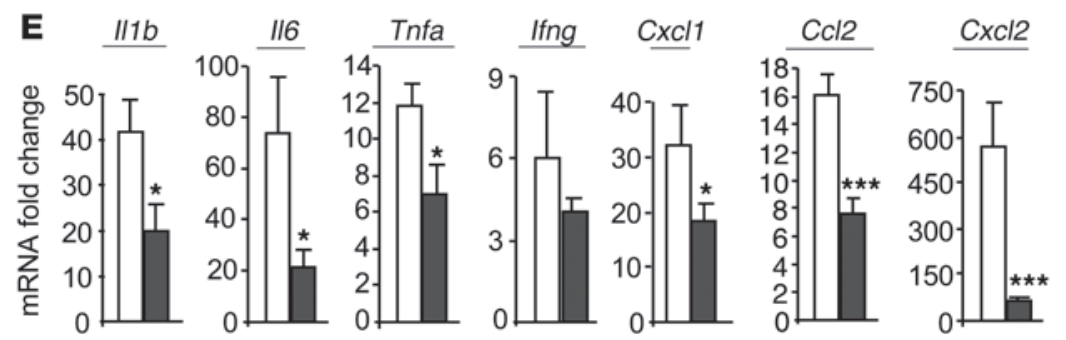

口CD98 $8^{\mathrm{H} / \mathrm{+}}$

- CD98 $8^{\mathrm{t} /+}$ VilCre

Figure 10

IEC-specific CD98 downregulation reduces colonic tumorigenesis in a mouse model of CAC. (A) CAC induction protocol. Mice were intraperitoneally injected with AOM and maintained for 7 days, then subjected to 2 cycles of DSS treatment ( 1 cycle representing 8 days of $2.5 \%$ DSS followed

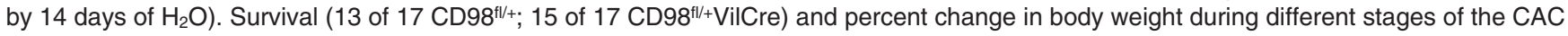
protocol are shown. (B) Representative colons at the end of the CAC protocol, with the number of tumors per mouse. (C) Tumor size was determined using a dissecting microscope. Size distribution of tumors is shown $\left(n=13\right.$ [CD98 $\left.{ }^{f / /+}\right] ; 15$ [CD98 $\left.\left.8^{\mathrm{fl}+\mathrm{V}} \mathrm{VilCre}\right]\right)$. (D) Representative H\&E-stained colonic tumors at the end of the CAC protocol. Scale bars: $100 \mu \mathrm{m}$. (E) Colonic cytokine/chemokine mRNA levels, quantified by qRT-PCR ( $n=9$ per group). Data are from 1 experiment repeated twice with similar results. ${ }^{*} P<0.05,{ }^{\star \star} P<0.005,{ }^{* \star} P<0.001$ vs. DSS-treated CD98 $8^{\mathrm{t} /+}$.

may result in abnormal basal intestinal phenotype and contribute to increased intestinal permeability. Our analysis of cytokine gene expression does not directly provide mechanistic explanations, but highlights important differences in the inflammatory profile of mice in which CD98 expression has been genetically manipulated. Intuitively, full elucidation of the mechanisms leading to cytokine dysregulation in $\mathrm{Tg}$ mice would require extensive characterization of their immune profile and the regulation of the development and activation of different immune cell populations.
Induction of cytokines and chemokines during inflammation, which enhances the proliferation and survival of IECs, is considered to be one of the major tumor-promoting mechanisms (3). Using genetic and pharmacological tools, inflammatory cytokines such as IL-6 (27) and TNF- $\alpha$ (28) have been increasingly shown to be crucial for CAC development and growth. Here, by challenging mice to a CAC model that combined AOM-induced carcinogenesis and DSS-induced chronic inflammation, we showed for the first time to our knowledge that IEC-specific CD98 overexpression enhanced 
A
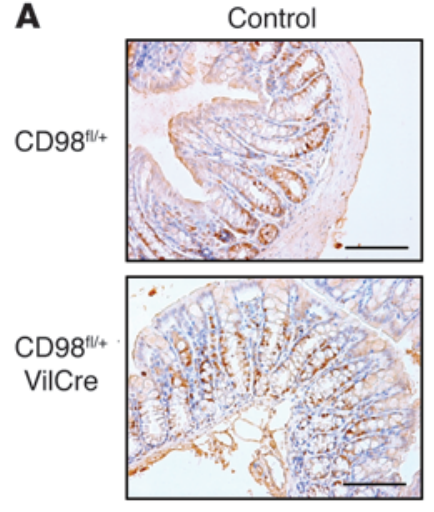

B

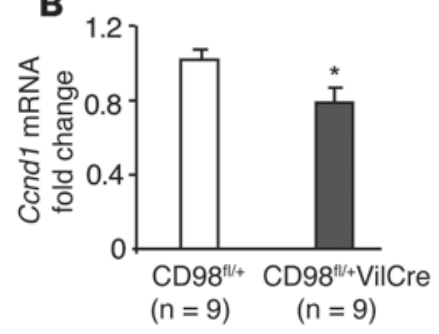

C

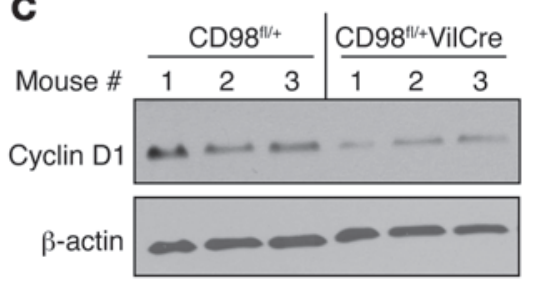

Figure 11

IEC-specific CD98 downregulation decreases cell proliferation during CAC development. Mice were subjected to the CAC protocol in Figure 10A. (A) Ki67 immunohistochemical staining of colonic sections from untreated (control) and AOM/DSS-treated mice. Scale bars: $100 \mu \mathrm{m}$. Colonic Ccnd1 mRNA and cyclin D1 protein levels were assessed by qRT-PCR (B; $n=9$ per group) and WB (C), respectively. Data are from 1 experiment repeated twice with similar results. ${ }^{*} P<0.05$ vs. $\mathrm{CD} 98^{\mathrm{fl} /+}$.

colonic tumor incidence and growth by stimulating inflammationinduced IEC proliferation and production of proinflammatory cytokines/chemokines, including the critical regulators IL- 6 and TNF- $\alpha$. In a chronic colitis-induced carcinogenesis model, wherein AOM-induced tumor initiation was omitted and chronic inflammation was the determinant for tumorigenesis, IEC-specific CD98 overexpression also enhanced the inflammatory responses during early and late states of tumorigenesis development, thereby promoting tumor incidence and growth. These consistent findings are strongly indicative of a role for IEC-specific CD98 overexpression in linking inflammation to tumorigenesis.

Furthermore, we showed that the CD98-overexpressing molecules were physiologically associated with $\beta_{1}$-integrin in IECs of Tg mice, affecting integrin-mediated FAK activation. Previously, $\beta_{1}$-integrin has been shown to play a crucial role in intestinal homeostasis (29) and tumorigenesis in mice (30). Increasing evidence has shown the importance of the integrin-FAK signaling linkage in the development of tumorigenesis (31). Indeed, the interaction of CD98 with $\beta_{1}$-integrin was previously shown to be required for FAK activationdependent cellular transformation in vitro (32). Thus, an increase in CD98/ $\beta_{1}$-integrin interaction complexes, which is accompanied by enhanced FAK activation, could also be an important mechanism underlying the tumorigenic activity of IEC-specific CD98 overexpression. It is of interest to note that although activation of Akt signaling was increased in jejunal enterocytes of $\mathrm{Tg}$ versus WT mice, no significant difference was observed in colonic enterocytes, which could be due to the lower expression of hCD98 in the colon compared with that in the jejunum. In contrast, increased Mendelian ratio at birth of $\mathrm{CD} 98^{\mathrm{f} / \mathrm{fl}} \mathrm{VilCre}$ mice with IEC-specific CD98 ablation was unexpected. This indicates that CD98 is crucial for intestinal homeostasis and gut development. Unlike these mice, $\mathrm{CD} 98^{\mathrm{fl} /+}$ VilCre mice with IEC-specific CD98 downregulation were born at the normal Mendelian ratio and displayed normal intestinal homeostasis. However, IEC-specific CD98 downregulation was sufficient to efficiently attenuate susceptibility of mice to DSS-induced colitis and CAC development. The reductive effect on tumor incidence and tumor size during CAC development of IECspecific CD98 downregulation was evidenced by its capability to attenuate inflammation-associated cytokine/chemokine production and IEC proliferation, consistent with the mechanism underlying tumorigenic activity of IEC-specific CD98 overexpression.

In summary, our findings suggest that intestinal CD98 is a critical molecule in regulating homeostatic and innate immune responses in the gut. Thus, we believe CD98 represents a potential therapeutic target for prevention and treatment of inflammatory intestinal diseases, such as IBD and CAC. We propose that maintaining a low level of CD98 expression in IECs during inflammation could have beneficial effects on preventing mucosal barrier disruption, tissue damage, and tumor development.

\section{Methods}

Association of SLC3A2 polymorphisms with IBD. The cohort of IBD patients and ethnically matched controls from Sweden has been previously described in detail (34-37). For the specific purpose of this study, $618 \mathrm{CD}$ patients, 630 UC patients, and 630 blood donor controls have been included. Diagnosis of IBD (i.e., CD or UC) was assessed in all cases according to established 
clinical criteria, including endoscopic, radiological, and histopathologic examination. Informed consent was obtained from all patients and controls, and the study protocol was approved by the local Swedish ethical committees of the Medical Faculties of Uppsala University (Uppsala, Sweden) and Karolinska Institute (Stockholm, Sweden).

To maximize the chances to detect association, 16 SLC3A2 SNPs were selected based on their tagging properties ( $r^{2}$ cutoff, 0.8 ; minor allele frequency cutoff, 0.2 ) and the highest potential to affect gene function and expression. This resulted in the inclusion of 5 tag SNPs (rs12804553, rs2282477, rs4726, rs12794763, and rs489381), 3 coding SNPs (rs3015957 [Pro79Arg], rs1140538 [Gly346Ala], and rs7944051 [Ser415Pro]), 6 SNPs mapping within 2 alternative predicted promoters (rs60101666, rs56686759, rs56765672, rs7126772, rs12274689, and rs11548756), and 2 SNPs contained within an enhancer element previously described (rs2070870 and rs73487879; ref. 38). Genotyping was performed on genomic DNA from all individuals using MALDI-TOF mass spectrometry, based on allele-specific primer extension with the iPLEX chemistry (Sequenom Inc.). Average genotyping success rate was $99.8 \%$, and no marker deviated significantly from expected Hardy-Weinberg equilibrium (cutoff, $P=0.05$ ). 8 SNPs (rs56686759, rs56765672, rs7126772, rs12274689, rs3015957, rs73487879, rs1140538, and rs7944051) proved monomorphic in the studied population and were therefore excluded from the statistic analyses. Linkage disequilibrium (LD) and haplotype block structure (solid spine of LD), as well as single marker and haplotypic associations, were investigated in Haploview 4.1 (http:// www.broad.mit.edu/mpg/haploview). SLC2A3 associations were tested on the entire sample (IBD vs. controls) and on subsets of patients grouped according to IBD subtype (CD vs. controls and UC vs. controls).

Mouse generation. To generate mice overexpressing CD98 specifically in IECs, the mouse villin promoter was used to drive transcription of the SLC3A2 gene, which encodes CD98, following an approach previously described (39). The full-length hCD98 cDNA $(10,40)$ was cloned into the $B s i W I / M l u I$ sites of the pBS KS Villin MES vector (39). The Tg DNA digested with SalI was purified and injected into the pronuclei of fertilized ova of FVB/N mice. Tg-carrying mice were identified by PCR analysis of tail genomic DNA with primers specific for the Tg: forward, 5'-GGCTGTGATAGCACACAGGA-3'; reverse, 5'-CCTTGGACAGGCCCGTGAACTTA-3'. Stable hCD98 Tg lines were established by backcrossing Tg-carrying founder mice with FVB/N mice (The Jackson Laboratory). hCD98-Villin Tg mice were identified among offspring, and IEC-specific hCD98 expression in $\mathrm{Tg}$ mice was examined by RT-PCR and WB. 3 different $\mathrm{Tg}$ founder lines were generated and tested with similar results.

CD $98^{\mathrm{fl} / \mathrm{fl}}$ mice were generated by flanking exons 1 and 2 of the Slc $3 \mathrm{a} 2$ alleles with loxP sites (Supplemental Methods). CD98 ${ }^{\mathrm{fl} /+}$ mice congenically rebred to $\mathrm{C} 57 \mathrm{BL} / 6$ for 8 generations were interbred to select homozygous $\mathrm{CD} 98^{\mathrm{f} / / \mathrm{fl}}$ mice. $\mathrm{CD} 98^{\mathrm{fl} / \mathrm{fl}}$ mice were maintained to cross with VilCre mice (JAX B6.SJL-Tg[Vil-Cre]997Gum/J, stock no. 004586; The Jackson Laboratory). The resulting offspring were genotyped by PCR of tail genomic DNA for expression of the floxed Slc3a2 allele and VilCre Tg. Cre-mediated recombination of the floxed Slc3a2 allele was verified by PCR of genomic DNA extracted from isolated IECs using a combination of 3 primers: 5'CreDel-S1, 5'-GGTTTTGGTTCTCAGGTAGG-3'; 3'CreDel-AS1, $5^{\prime}$-GCTGCTTGTGTTGTGCTGAG-3'; and the Cre-recombination primer, 5'-GGGATAGACGGGAGTATTC-3'. Screening was made based on the presence or absence of 3 PCR products: WT (301 bp), mutant (413 bp), and the $356-\mathrm{bp}$ product indicating recombination of the floxed $S l c 3 a 2$ allele.

All mice were maintained in standard cages and were allowed standard chow and tap water ad libitum. All animal procedures were approved by the Emory University Institutional Animal Care and Use Committee.

Colitis and tumor induction. Colitis was induced in WT and Tg littermates by oral administration of $3.5 \%$ (w/v) DSS (36-50 kDa; MP Biomedicals) in drinking water. For CD $98^{\mathrm{fl} /+}$ and $\mathrm{CD} 98^{\mathrm{fl} /+} \mathrm{VilCre}$ littermates, colitis was induced with $3 \%$ DSS. Age- and sex-matched littermates receiving drinking water were served as controls. Mice were weighed daily. Clinical and histological scores were assessed at the end of DSS treatment.

CAC was induced as previously described (26), with some modifications. Mice were intraperitoneally injected with AOM (10 mg/ $\mathrm{kg}$ body weight) and maintained on regular diet and water for 5 days (WT and Tg mice) or 7 days (CD98 $8^{\mathrm{fl} /+}$ and $\mathrm{CD} 98^{\mathrm{fl} /+}$ VilCre mice). Mice were then subjected to 2 cycles of DSS treatment, in which each cycle consisted of $2.5 \%$ DSS for 7 days (WT and $\mathrm{Tg}$ mice) or 8 days (CD98 $8^{\mathrm{f} /+}$ and CD98 $8^{\mathrm{fl} /+}$ VilCre mice) followed by a 14-day recovery period with regular water.

In the chronic colitis-induced CAC model, which lacks the use of a carcinogen, mice were subjected to 5 cycles of DSS treatment (with 1 cycle representing 7 days of $2.5 \%$ DSS followed by 14 days of $\mathrm{H}_{2} \mathrm{O}$ ). Colonic tumors were counted and measured using a dissecting microscope.

Clinical activity score. Assessment of body weight loss, stool consistency, and the presence of occult/gross blood by a guaiac test (Hemoccult Sensa; Beckman Coulter) was done daily for each mouse. Body weight change was scored as follows: 0 , no change; $1,1 \%-5 \%$ weight loss; $2,5 \%-10 \%$ weight loss; $3,10 \%-20 \%$ weight loss; $4,>20 \%$ weight loss. Stool character was scored as follows: 0 , normal; 1 , soft with well-formed pellets; 2 , soft without pellets; 4, diarrhea. Occult blood was scored as follows: 0 , no blood; 2 , positive Hemoccult (Beckman Coulter); 4, gross bleeding. These scores were added to generate a clinical activity score ranging from 0 to 12 .

Histology. Distal colonic sections were fixed in $10 \%$ formalin and embedded in paraffin. $5-\mu \mathrm{m}$ sections were stained with H\&E. Photomicrographs were taken using a Nikon Eclipse TS100 microscope.

Histological assessment of colitis. H\&E-stained colonic sections were coded for blind microscopic assessment of inflammation (i.e., DSS-induced colitis). Histological scoring was based on 3 parameters. Severity of inflammation was scored as follows: 0 , rare inflammatory cells in the lamina propria; 1 , increased numbers of granulocytes in the lamina propria; 2 , confluence of inflammatory cells extending into the submucosa; 3 , transmural extension of the inflammatory infiltrate. Crypt damage was scored as follows: 0 , intact crypts; 1 , loss of the basal one-third; 2, loss of the basal two-thirds; 3 , entire crypt loss; 4, change of epithelial surface with erosion; 5 , confluent erosion. Ulceration was scored as follows: 0 , absence of ulcer; 1,1 or 2 foci of ulcerations; 2 , 3 or 4 foci of ulcerations; 3 , confluent or extensive ulceration. Values were added to give a maximal histological score of 11 .

Immunohistochemistry. 5- $\mu \mathrm{m}$ paraffin-embedded tissue sections were deparaffinized in xylene, incubated in $3 \%$ hydrogen peroxide in methanol for 30 minutes, rehydrated in ethanol gradient, and then treated with $10 \mathrm{mM}$ sodium citrate buffer ( $\mathrm{pH}$ 6.0) containing $0.05 \%$ Tween 20 at $120^{\circ} \mathrm{C}$ for 10 minutes in a pressure cooker. Sections were blocked with $2 \%$ nonfat dry milk and $0.01 \%$ Tween 20 in PBS for 1 hour at $37^{\circ} \mathrm{C}$, and then incubated with anti-Ki67 (Novocastra) or anti-human CD98 antibody (Santa Cruz) overnight at $4{ }^{\circ} \mathrm{C}$. After washes with PBS containing $0.01 \%$ Tween 20 , sections were treated with appropriate biotinylated secondary antibodies for 30 minutes at $37^{\circ} \mathrm{C}$, and color development was performed using the Vectastain $\mathrm{ABC}$ kit (Vector Laboratories). Sections were then counterstained with hematoxylin, dehydrated, and coverslipped. Images were acquired using a Zeiss Axioskop 2 plus microscope (Carl Zeiss MicroImaging) equipped with an AxioCam MRc5 CCD camera (Carl Zeiss).

Electron microscopy. Intestine tissues were dissected into 1- to 2-mm cubes while immersed in $2.5 \%$ glutaraldehyde buffered with $0.1 \mathrm{M}$ sodium cacodylate ( $\mathrm{pH}$ 7.2). Samples were stored in the same fixative overnight at $4{ }^{\circ} \mathrm{C}$. Samples were then washed with the same buffer and postfixed in $1 \%$ buffered osmium tetroxide, dehydrated through a graded ethanol series to $100 \%$, and embedded in Eponate 12 resin (Ted pella Inc.). Ultrathin sec- 
tions were cut on a Leica UC6rt ultramicrotome (Leica Microsystems) at $70-80 \mathrm{~nm}$ and counterstained with $4 \%$ aqueous uranyl acetate and $2 \%$ lead citrate. Sections were examined using a Hitachi H-7500 transmission electron microscope (Hitachi High Technologies of America Inc.) equipped with a Gatan BioScan CCD camera.

Microarray analysis. Total RNAs extracted from jejunum and colon of WT, $\mathrm{Tg}$, and Tg-HE mice using the RNeasy Mini Kit (Qiagen) were pooled from 4 littermates per group. All RNA samples were DNase treated (Rnase-free; Invitrogen), subjected to quality control analysis, labeled using the TotalPrep RNA labeling kit (Ambion), and hybridized to the Mouse WG-6 v2 Expression BeadChip (Illumina) that targets a total of 45,281 transcripts. Arrays were scanned on Illumina BeadStation 500. After data normalization, genes with uniformly low expression (detection $P \geq 0.05$ ) were removed from further analyses, and data were analyzed using Significance Analysis of Microarrays (SAM 2.23) software. Genes with at least 1.5-fold change relative to WT mice were selected and annotated using the DAVID Bioinformatics Resources 2008 (23). Microarray data were deposited in ArrayExpress (accession no. E-MTAB-486; http://www.ebi.ac.uk/cgi-bin/ microarray/magetab.cgi).

In vivo permeability assay, IEC isolation, TUNEL assay, WB, RT-PCR and qRT-PCR, Southern blot, ELISA, and MPO assay. See Supplemental Methods.
Statistics. Values are expressed as mean \pm SEM. Statistical analysis was performed using unpaired 2-tailed Student's $t$ test by InStat (version 3.06; GraphPad). A $P$ value less than 0.05 was considered significant.

\section{Acknowledgments}

This work was supported by grants from the Department of Veterans Affairs and the National Institute of Diabetes and Digestive and Kidney Diseases (R24-DK-064399 center grant, R01DK071594 to D. Merlin, and RO1-DK-55850 to S.V. Sitaraman) as well as by a research fellowship award from the Crohn's and Colitis Foundation of America (to G. Dalmasso). We are grateful to Vincent Yang (Emory University) for helping with VilCre mice and to Amr Ghaleb, Mandayam Nandan, and Tracy Obertone (Emory University) for technical advice.

Received for publication August 4, 2010, and accepted in revised form January 26, 2011.

Address correspondence to: Hang Thi Thu Nguyen, Emory University, Department of Medicine, 615 Michael Street, Atlanta, Georgia 30322, USA. Phone: 404.727.6246; Fax: 404.727.5767; E-mail: hnguye9@emory.edu.
1. Weir HK, et al. Annual report to the nation on the status of cancer, 1975-2000, featuring the uses of surveillance data for cancer prevention and control. J Natl Cancer Inst. 2003;95(17):1276-1299.

2. Coussens LM, Werb Z. Inflammation and cancer. Nature. 2002;420(6917):860-867.

3. Karin M, Lawrence T, Nizet V. Innate immunity gone awry: linking microbial infections to chronic inflammation and cancer. Cell. 2006;124(4):823-835.

4. Terzic J, Grivennikov S, Karin E, Karin M. Inflammation and colon cancer. Gastroenterology. 2010; 138(6):2101-2114.e5

5. Yan Y, Vasudevan S, Nguyen HT, Merlin D. Intestinal epithelial CD98: an oligomeric and multifunctional protein. Biochim Biophys Acta. 2008;1780(10):1087-1092.

6. Deves R, Boyd CA. Surface antigen CD98(4F2): not a single membrane protein, but a family of proteins with multiple functions. J Membr Biol. 2000;173(3):165-177.

7. Kehrl JH, Fauci AS. Identification, purification, and characterization of antigen-activated and antigen-specific human B lymphocytes. J Exp Med. 1983;157(5):1692-1697.

8. Fenczik CA, Sethi T, Ramos JW, Hughes PE, Ginsberg MH. Complementation of dominant suppression implicates CD98 in integrin activation. Nature. 1997;390(6655):81-85.

9. Feral CC, Nishiya N, Fenczik CA, Stuhlmann H, Slepak M, Ginsberg MH. CD98hc (SLC3A2) mediates integrin signaling. Proc Natl Acad Sci U S A. 2005;102(2):355-360.

10. Merlin D, et al. CD98-mediated links between amino acid transport and beta 1 integrin distribution in polarized columnar epithelia. J Biol Chem. 2001;276(42):39282-39289.

11. Dixon WT, Sikora LK, Demetrick DJ, Jerry LM. Isolation and characterization of a heterodimeric surface antigen on human melanoma cells and evidence that it is the 4F 2 cell activation/proliferation molecule. Int J Cancer. 1990;45(1):59-68.

12. Esteban F, Ruiz-Cabello F, Concha A, Perez Ayala M, Delgado M, Garrido F. Relationship of 4F2 antigen with local growth and metastatic potential of squamous cell carcinoma of the larynx. Cancer. 1990;66(7):1493-1498.

13. Garber ME, et al. Diversity of gene expression in adenocarcinoma of the lung. Proc Natl Acad SciUS A. 2001;98(24):13784-13789.

14. Esseghir $S$, et al. Identification of transmembrane pro- teins as potential prognostic markers and therapeutic targets in breast cancer by a screen for signal sequence encoding transcripts. J Pathol. 2006;210(4):420-430.

15. Prager GW, et al. CD98hc (SLC3A2), a novel marker in renal cell cancer. Eur J Clin Invest. 2009;39(4):304-310.

16. Kaira K, et al. l-type amino acid transporter 1 and CD98 expression in primary and metastatic sites of human neoplasms. Cancer Sci. 2008; 99(12):2380-2386.

17. Kucharzik T, et al. Activation of epithelial CD98 glycoprotein perpetuates colonic inflammation. Lab Invest. 2005;85(7):932-941.

18. Fais S, Pallone F. Ability of human colonic epithelium to express the 4F2 antigen, the common acute lymphoblastic leukemia antigen, and the transferrin receptor. Studies in inflammatory bowel disease and after in vitro exposure to different stimuli. Gastroenterology. 1989;97(6):1435-1441.

19. Yan Y, Dalmasso G, Sitaraman S, Merlin D. Characterization of the human intestinal CD98 promoter and its regulation by interferon-gamma. Am J Physiol Gastrointest Liver Physiol. 2007;292(2):G535-G545.

20. Schreiber S, MacDermott RP, Raedler A, Pinnau R, Bertovich MJ, Nash GS. Increased activation of isolated intestinal lamina propria mononuclear cells in inflammatory bowel disease. Gastroenterology. 1991;101(4):1020-1030.

21. Nguyen HT, et al. MicroRNA-7 modulates CD98 expression during intestinal epithelial cell differentiation. J Biol Chem. 2010;285(2):1479-1489.

22. Nakamura E, et al. 4F2 (CD98) heavy chain is associated covalently with an amino acid transporter and controls intracellular trafficking and membrane topology of 4F2 heterodimer. J Biol Chem. 1999;274(5):3009-3016.

23. Dennis G Jr, et al. DAVID: Database for annotation, visualization, and integrated discovery. Genome Biol. 2003;4(5):P3.

24. Hynes RO. Integrins: bidirectional, allosteric signaling machines. Cell. 2002;110(6):673-687.

25. Sartor RB. Mechanisms of disease: pathogenesis of Crohn's disease and ulcerative colitis. Nat Clin Pract Gastroenterol Hepatol. 2006;3(7):390-407.

26. Greten FR, et al. IKKbeta links inflammation and tumorigenesis in a mouse model of colitis-associated cancer. Cell. 2004;118(3):285-296.

27. Grivennikov S, et al. IL-6 and Stat 3 are required for survival of intestinal epithelial cells and development of colitis-associated cancer. Cancer Cell. 2009;15(2):103-113.
28. Popivanova BK, et al. Blocking TNF-alpha in mice reduces colorectal carcinogenesis associated with chronic colitis. J Clin Invest. 2008;118(2):560-570.

29. Jones RG, et al. Conditional deletion of beta 1 integrins in the intestinal epithelium causes a loss of Hedgehog expression, intestinal hyperplasia, and early postnatal lethality. J Cell Biol. 2006; 175(3):505-514.

30. White DE, et al. Targeted disruption of beta1-integrin in a transgenic mouse model of human breast cancer reveals an essential role in mammary tumor induction. Cancer Cell. 2004;6(2):159-170.

31. McLean GW, Carragher NO, Avizienyte E, Evans J, Brunton VG, Frame MC. The role of focal-adhesion kinase in cancer - a new therapeutic opportunity. Nat Rev Cancer. 2005;5(7):505-515.

32. Henderson NC, et al. CD98hc (SLC3A2) interaction with beta 1 integrins is required for transformation. J Biol Chem. 2004;279(52):54731-54741.

33. Tsumura $\mathrm{H}$, et al. The targeted disruption of the CD98 gene results in embryonic lethality. Biochem Biophys Res Commun. 2003;308(4):847-851.

34. Bresso F, et al. Potential role for the common cystic fibrosis DeltaF508 mutation in Crohn's disease. Inflamm Bowel Dis. 2007;13(5):531-536.

35. D'Amato M, et al. Neuropeptide s receptor 1 gene polymorphism is associated with susceptibility to inflammatory bowel disease. Gastroenterology. 2007;133(3):808-817.

36. Zucchelli $\mathrm{M}$, et al. PepT1 oligopeptide transporter (SLC15A1) gene polymorphism in inflammatory bowel disease. Inflamm Bowel Dis. 2009; 15(10):1562-1569.

37. McGovern DP, et al. Genome-wide association identifies multiple ulcerative colitis susceptibility loci. Nat Genet. 2010;42(4):332-337.

38. Karpinski BA, Yang LH, Cacheris P, Morle GD, Leiden JM. The first intron of the 4F2 heavy-chain gene contains a transcriptional enhancer element that binds multiple nuclear proteins. Mol Cell Biol. 1989;9(6):2588-2597.

39. Pinto D, Robine S, Jaisser F, El Marjou FE, Louvard D. Regulatory sequences of the mouse villin gene that efficiently drive transgenic expression in immature and differentiated epithelial cells of small and large intestines. J Biol Chem. 1999;274(10):6476-6482.

40. Nguyen HT, Dalmasso G, Yan Y, Obertone TS, Sitaraman SV, Merlin D. Ecto-phosphorylation of CD98 regulates cell-cell interactions. PLoS One. 2008;3(12):e3895 